\title{
Calibration of an extended Eddy Viscosity Turbulence Model using Uncertainty Quantification
}

\author{
Gokul Subbian, Ana Carolina Botelho e Souza*, Rolf Radespiel ${ }^{\dagger}$, \\ Elmar Zander and Thilo Moshagen \\ Technische Universität Braunschweig, 38108 Braunschweig, Germany \\ Noemi Friedman ${ }^{\S}$ \\ Institute for Computer Science and Control (SZTAKI), Budapest, Hungary \\ Matteo Moioli ${ }^{\text {II }}$, Christian Breitsamter" \\ Technische Universität München, 85748 Garching, Germany \\ Kaare Sørensen** \\ Airbus Defense and Space, Manching , Germany
}

The present work aims at improving the performance of the eddy viscosity based MenterSST turbulence model for complex aircraft flows by adding physical extensions to the $\omega$ length scale equation along with calibration aided by Uncertainty Quantification methods. Three different extensions based on rotation and curvature correction, wake flow correction and irrotational strain correction are added to the original model, resulting in a parametric model, which is then calibrated using Bayesian Updates. A series of test cases is employed to assess the model performance, whereby delta wings with deflected flaps and vortical flows are the predominant target application.

\section{Nomenclature}

$\begin{array}{ll}U & =\text { Velocity, } \mathrm{m} / \mathrm{s} \\ \tau_{i j} & =\text { Viscous stress component, } \mathrm{N} / \mathrm{m}^{2} \\ k & =\text { Turbulent kinetic energy, } \mathrm{m}^{2} / \mathrm{s}^{2} \\ \kappa & =\text { Von Karman Constant } \\ p & =\text { Pressure, Pa } \\ \delta_{i j} & =\text { Kronecker symbol } \\ \omega & =\text { Vorticity, } \mathrm{s}^{-1} \\ Q & =\text { Second invariant, } \mathrm{s}^{-2} \\ P_{i j} & =\text { Production tensor, } \mathrm{m}^{2} / \mathrm{s}^{3} \\ v & =\text { Kinematic Viscocity, } \mathrm{m}^{2} / \mathrm{s} \\ \varepsilon & =\text { Dissipation rate, } \mathrm{m}^{2} / \mathrm{s}^{2} \\ \mu & \left.=\text { Dynamic viscosity. } \mathrm{kg}^{2} / \mathrm{ms}\right) \\ M & =\text { Mach number } \\ \omega & =\text { Specific dissipation rate, } 1 / \mathrm{s} \\ R e & =\text { Reynolds number } \\ \alpha & =\text { Angle of attack, deg } \\ C_{l} & =\text { Lift coefficient }\end{array}$

\footnotetext{
${ }^{*}$ Research Assistant, Institute of Fluid Mechanics, TU Braunschweig.

${ }^{\dagger}$ Professor and Head of Institute, Institute of Fluid Mechanics, TU Braunschweig.

$\doteqdot$ Institute of Scientific Computing, TU Braunschweig.

$\S$ Research fellow

"Research Assistant, Chair of Aerodynamics and Fluid Mechanics, TU München

"Professor, Chair of Aerodynamics and Fluid Mechanics, TU München

** Engineer, Airbus Defense and Space, Manching, Germany
} 


$\begin{array}{ll}C_{d} & =\text { Drag coefficient } \\ C_{f} & =\text { Skin friction coefficient } \\ C_{p} & =\text { Coefficient of pressure } \\ \eta & =\text { Wing-Spanwise position coefficient, Root-0 and Tip-1 } \\ \frac{u, v, w}{u^{\prime 2}, \overline{v^{\prime 2}}, \overline{w^{\prime 2}}} & =\text { X,Y,Z velocity components } \\ -\rho \overline{u_{i}^{\prime} u_{j}^{\prime}} & =\text { Fluctuating velocity components } \\ x, y, z & =\text { Cartesian co-ordinates } \\ y^{+} & =\text {Wall distance } \\ F 1, F 2 & =\text { Menter-SST blending functions } \\ v_{T} & =\text { Eddy viscocity } \\ \alpha & =\text { angle of attack } \\ \Omega & =\text { Absolute vorticity, } 1 / s\end{array}$

\section{Introduction}

$\mathrm{O}$ E of the commonly used flow simulation approaches for the purpose of computing external flows is the Reynolds Averaged Navier Stokes (RANS) equation method. The complex turbulence fluctuations in the flow field are captured by the underlying turbulence models. Generally, these turbulent fluctuations are represented by the Reynoldsstress tensor in the momentum equation. Different assumptions are used for modeling the Reynolds-stress tensor, which categorizes the type of the turbulence model used in the solver. One of the widely used is the Boussinesq assumption, which relates linearly the stress tensor to the velocity gradients by means of the turbulent viscocity. In the case of the two-equation eddy viscocity turbulence model (Menter-SST) which is used in this work, two transport equations are used to describe the transport of two scalars and the Boussinesq assumption is used for the Reynolds-stress tensor. Due to the simplification for the Reynolds-stress tensor, the turbulence model is not well suited for flows where stress and strain rates are not aligned or where a rapid redistribution among the stresses takes place. This is the case in vortical flow and in many separated flows. Neverthless, Menter-SST is the most widely used turbulence model for the computation of industrial flows due to its cost effectiveness in computational time and its robustness.

Several improvements of the Menter-SST model for better prediction capabilities have been suggested and implemented in the DLR-TAU code. Vortical correction methods have been implemented which take into account the effects of frame rotation to the production of Reynolds stresses, which was previously lacking in the original model [1]. The main objective of this work is to exploit the potentials of different correction methods for a two-equation model (Menter-SST) of turbulence. Therefore, three correction methods are investigated within this project: the vortex correction method, the wake flow correction method and a correction method that takes the effects of irrotational strains on turbulence into account. It has to be noted that the final model comprises all the three additional extensions together with the original model as a single extended variant of the Menter-SST model. Section III gives an overview of the individual extensions, section $\mathrm{V}$ provides the scope of the test cases involved in this work and section IV]describes the calibration process of the extended model.

\section{Extensions to Eddy Viscosity Model}

\section{A. Motivation}

Vortical flows are observed in internal flow fields, e.g. for increasing the combustion efficiency through swirl injectors. Predominant occurrence of vortical flows takes place in external aerodynamics such as wing tip vortices and delta wings. Vortices are also utilized in high lift devices such as nacelle strakes to increase the lift coefficient. The characteristics of the vortices strongly influence the performance of the lifting device and flow domain. In the case of low aspect ratio delta wings, the vortex sheet separating at the swept leading edge separation causes a local low pressure region on the suction side which increases the overall lift [2]. The generated vortex sheet is highly influenced by the pressure gradients in its vicinity. While vortices will diffuse in the flow field under normal conditions, vortex breakdown is encountered in some special cases [3]. The location and mode of breakdown depends on various parameters such as adverse pressure gradients, type of delta wing planforms, angle of attack, sweep angle. Blowing or suction over the profile were also found to affect the breakdown location, as well as flaps and other control devices which alter the effective camber of the profile are some of the factors. In many cases, the axial velocity of the vortex core is well above 
the surrounding velocity of the freestream conditions at the origin of vortex flow. At the point of vortex breakdown, this jet like vortex flow breaks down into smaller structures experiencing abrupt decelerations, which eventually become wake like flow [4]. This is a highly turbulent phenomenon. The pitching moment characteristics of the delta wings are influenced by the breakdown over the wing planforms [5]. High pressure fluctuations are created over the suction side of the delta wings due to breakdown, thereby affecting the operating envelope of low aspect ratio aircraft [6] . Therefore, it is of high importance to understand the characteristics and occurrence of vortex breakdown. Accurate prediction of vortex flows and breakdown is a challenging requirement in the field of turbulence modeling.

Delta wings are suitable test cases for vortical flows due to the generation of stable vortices in a relatively uncomplicated manner. They facilitate simpler experimental setup and are also easier to model numerically, thereby the main emphasize can be given to the capturing of vortical flow field in experiments and modeling in numerical studies. In the case of delta wings with sharp leading edge profile, the flow separation takes place along the entire leading-edge at some angle of attack. As the bluntness of the leading edge increases, the onset of separation recedes progressively downstream along the leading edge. Discrete vortices are formed along the leading edge due to the separation and they roll together to form a single larger vortex sheet [7]. Based on the pressure gradients over the suction side, occurrences of secondary as well as tertiary vortices have been observed.

RANS based computational approaches are important for these kind of complex aeronautical flows, since direct numerical and large eddy simulations require much larger computational resources and time. Various types of turbulence models are available in RANS approach, however no single turbulence model, which can be satisfactorily used for all kind of applications exists. One equation eddy viscosity models are relatively simple and robust. Reynolds Stress Models are often less robust, although their prediction capabilities are superior in general. Two equation eddy viscosity models offer a good compromise between solution accuracy and complexity. In specific, the Menter-SST [8] turbulence model is widely used in external aerodynamic flow fields. However, a primary deficiency with the eddy viscosity models appears in their inability to account for turbulent anisotropies in rotating and separated flows. From existing literature, different model extensions are available to the Menter-SST turbulence model, which are proposed to offer better accuracy in the case of rotational flow problems.

\section{B. Development and Implementation}

\section{Menter-SST Model}

The Menter-Shear Stress Transport model is constituted of two different two equation models. In the near wall region, the model is based on the original $\mathrm{k}-\omega$ model and in the outer boundary layer it is based upon the k- $\epsilon$ model. Since this is a well established model, only the two transport equations [8] are provided here as an overview.

$$
\begin{gathered}
\frac{D \rho k}{D t}=\tau_{i j} \frac{\partial u_{i}}{\partial x_{j}}-\beta^{*} \rho \omega k+\frac{\partial}{\partial x_{j}}\left[\left(\mu+\sigma_{k} \mu_{t}\right) \frac{\partial k}{\partial x_{j}}\right] \\
\frac{D \rho \omega}{D t}=\frac{\gamma}{v_{t}} \tau_{i j} \frac{\partial u_{i}}{\partial x_{j}}-\beta \rho \omega^{2}+\frac{\partial}{\partial x_{j}}\left[\left(\mu+\sigma_{\omega} \mu_{t}\right) \frac{\partial \omega}{\partial x_{j}}\right]+2\left(1-F_{1}\right) \rho \sigma_{\omega 2} \frac{1}{\omega} \frac{\partial k}{\partial x_{j}} \frac{\partial \omega}{\partial x_{j}}
\end{gathered}
$$

Boussinesq assumption:

$$
-\bar{\rho} \tilde{u}_{i} u_{j}^{\prime \prime}=\mu_{t}\left(\frac{\partial \tilde{u_{i}}}{\partial x_{j}}+\frac{\partial \tilde{u_{j}}}{\partial x_{i}}-\frac{2}{3} \frac{\partial \tilde{u_{k}}}{\partial x_{k}} \delta_{i j}\right)-\frac{2}{3} \bar{\rho} k \delta_{i j}
$$

\section{SARC}

One of the predominant drawbacks of the Menter-SST model is the low accuracy involving system rotation and streamline curvatures. An initial approach to address this weakness is the introduction of a term called "gyroscopic stability effect" by Knight and Saffman [9]. This term includes the angular velocity of the principal axes of the mean rate of strain tensor, which was added to the transport equations. Spalart-Shur correction was proposed using a similar principle to that of the Knight and Saffman approach. One of the main advantages of the Spalart-Shur approach is the availability of an explicit formula, simplifying the numerical implementation process for three-dimensional flows [10]. The correction approach is Galilean invariant [11]. Initially, it was applied to the Spalart-Allmaras one equation turbulence model. Menter adapted this curvature correction approach to the standard Menter-SST model. The original 
empirical function defined through the Spalart-Shur correction is given by [10]:

$$
f_{\text {rotation }}=\left(1+c_{r 1}\right) \frac{2 r^{*}}{1+r^{*}}\left[1-c_{r 3} \tan ^{-1}\left(c_{r 2} \tilde{r}\right)\right]-c_{r 1}
$$

The above function was originally multiplied with the production terms in the transport equations of the Spalart-Allmaras eddy viscosity turbulence model. In the case of the Menter-SST model, the production terms are based on the strain rate tensor $S$ whereas that of the Spalart-Allmaras model is based on the vorticity tensor $\Omega$ [12]. Since the production based on the strain rate tensor is typically higher than that of the vorticity tensor, Menter introduced a limiter to the function and adapted it for the Menter-SST model. The redefined function is given by,

$$
f_{r 1}=\max \left\{\min \left(f_{\text {rotation }}, 1.25\right), 0.0\right\}
$$

The limit value of 1.25 was based on the test conducted by Menter et al. [1] and the lower limit of 0 is added for the numerical stability purpose. The modified transport equations of $k$ and $\omega$ for the Menter-SST with the curvature correction approach is as follows [12]:

$$
\begin{gathered}
\frac{\partial(\rho k)}{\partial t}+\frac{\partial\left(\rho u_{j} k\right)}{\partial x_{j}}=P_{k} f_{r 1}-\beta^{*} \rho k \omega+\frac{\partial}{\partial x_{j}}\left[\mu_{e f} \frac{\partial k}{\partial x_{j}}\right] \\
\frac{\partial(\rho \omega)}{\partial t}+\frac{\partial\left(\rho u_{j} \omega\right)}{\partial x_{j}}=\alpha \frac{\rho P_{k}}{\mu_{t}} f_{r 1}-D_{\omega}+C d_{\omega}+\frac{\partial}{\partial x_{j}}\left[\mu_{e f} \frac{\partial \omega}{\partial x_{j}}\right]
\end{gathered}
$$

The values of the correction function constants $c_{r 1}, c_{r 2}$ and $c_{r 3}$ are 1,2 and 1 respectively.

\section{SAS}

It has been observed that relatively lower values of Reynolds stresses are predicted by Menter-SST model in flows involving separation and highly curved velocity profiles. To counteract this deficiency, either the levels of turbulence produced should be increased or the dissipation levels in these regions should be reduced. Menter proposed a Scale-Adaptive-Simulation (SAS) based model, which considers the local inhomogeneities in the flowfield [13]. It is based on a second velocity derivative term from the Rotta's $k-k L$ model. In the SST-SAS model, an additional term is added to the $\omega$ length scale equation. The advantages of using the SST-SAS model over the unsteady RANS had already been shown by Menter [13].

In this work, the model concept is implemented as in the previous works of Maduta et al. [14] and Cecora et al. [15] in Reynolds Stress Models. For the sake of simplicity, the additional term is referred to as SAS although no scale resolving simulations are modeled nor performed. The additional SAS term is used as a sink term in the dissipation equation and not as a source term as considered by Menter. In addition to increasing the production of turbulence in the specific regions, this term is attributed to stabilize the simulations, therby aiding in the convergence. The SAS term is given as follows:

$$
Q_{S A S}=\max \left[\rho \zeta_{2} K S^{2}\left(\frac{L}{L_{v k}}\right)^{2}-C \cdot \frac{2 \rho k}{\sigma_{\Phi}} \max \left(\frac{|\nabla \omega|^{2}}{\omega^{2}}, \frac{|\nabla k|^{2}}{k^{2}}\right), 0\right]
$$

The $Q_{S A S}$ term is added as a sink term in equation 2 The constant $\zeta_{2}$ has a value of 3.51 and the length scale ratios are given by:

$$
\begin{aligned}
& L=\frac{\sqrt{k}}{\left(c_{\mu}^{1 / 4} \cdot \omega\right)} \\
& L_{v k}=\frac{\kappa S}{\left|\nabla^{2} U\right|}
\end{aligned}
$$

\section{Irrotational Strains}

Another shortcoming of the Menter-SST model is the low accuracy in flows involving adverse pressure gradients. Hanjalić and Launder proposed a term to sensitize the dissipation equation to irrotational strains in the k- $\epsilon$ turbulence model [16]. Based on this previous work, Probst [17] developed and implemented a source term for the Reynolds Stress Model, accounting for adverse pressure gradients in the flowfield. The term is based on a streamline oriented coordinate system and in addition to the principal streamline direction, two independent normal directions are considered. The term developed by Probst is suitable for an $\epsilon$-based turbulence model, and it has been adapted to the $\omega$ length scale equation of the SST model. However this model extension is non Galilean invariant due to its dependency on streamline oriented coordinate system. Neverthless, this extension was used as a preliminary variant of sensitizing the model to 
the effects of irrotational strains and hence pressure gradients. The term which differentiates between the effects of irrotational and rotational straining is given as follows:

$$
S_{\omega}=-C_{\omega}^{*} \frac{\omega}{k}\left(\overline{u_{s}^{\prime 2}} \frac{\partial U_{s}}{\partial x_{s}}+\overline{u_{n 1}^{\prime 2}} \frac{\partial U_{n 1}}{\partial x_{n 1}}+\overline{u_{n 2}^{\prime 2}} \frac{\partial U_{n 2}}{\partial x_{n 2}}\right)
$$

The term $S_{\omega}$ is multiplied by density and added to the equation 2 The constant $C_{\omega}^{*}$ has a value of 0.58 . This is referred to as PG.

In order to enhance the model's performance in flows with large pressure gradients, the source term based on equation 11 in the length scale equation was considered as described in section III.B.4 During the implementation phase, this term led to significant improvements in several different test cases. However, due to its use of frame-dependent quantities, this formulation is not Galilean-invariant. The fulfillment of this criteria is essential for models devised to be employed e.g. in the turbomachinery and aero engine sectors, where the frame of reference is not fixed due to rotating components. This requirement supported the development of a new term, referred to as "Vorticity squared to Production of k difference", and hence V2Pkd.

The new formulation of the additional term is also based on the work of Hanjalić and Launder, although devised in a cartesian frame of reference. It takes into account the subtraction of a strain rate-dependent component from a vorticity-dependent component. The difference is then multiplied by a scalar coefficient that regulates the term's intensity. The term is formulated as follows:

$$
\begin{array}{r}
V 2 P k d=C_{\omega 4} \rho\left(W_{i j} W_{i j}-S_{i j} S_{i j}\right) \\
W_{i j}=\frac{1}{2}\left(\frac{\partial U_{i}}{\partial x_{j}}-\frac{\partial U_{j}}{\partial x_{i}}\right) \quad S_{i j}=\frac{1}{2}\left(\frac{\partial U_{i}}{\partial x_{j}}+\frac{\partial U_{j}}{\partial x_{i}}\right)
\end{array}
$$

where $W_{i j}$ and $S_{i j}$ are, respectively, the mean vorticity tensor and the mean strain rate tensor. $C_{\omega 4}$ is the term's coefficient. In equilibrium boundary layers $\left(P_{k} \sim \epsilon\right)$, the term is nullified because $W_{i j}$ and $S_{i j}$ have approximately the same magnitude thus its subtraction amounts to zero. On the other hand, in non-equilibrium flows, the predominance of its vorticity- and strain rate-components alternate and the sign of the term varies throughout the flow. When the V2Pkd term assumes a positive sign, it is interpreted as a source in the $\omega$-equation 2 leading to an increase in dissipation and subsequent decrease in turbulent stresses. In contrast, when the term is negative, it acts as a sink leading to the opposite effect, i.e. decrease in dissipation and increase in turbulent stresses.

\section{Initial Validation}

The Zero Pressure Gradient Flat Plate (ZPGFP) is a thin plate, which provides a very low thickness to chord ratio. The top half portion of the plate was simulated, using the symmetry condition along the axis. Farfield boundary condition was specified at a semi-circle with a radius of $10 \mathrm{~m}$ from the midpoint of the plate. The initial wall spacing used was $6.3 * 10^{-4} \mathrm{~mm}$. Hyperbolic as well as normal extrusions were used with a growth rate of 1.1. Based on the error estimation equations for the mesh convergence, the uncertainty in the skin friction coefficient computed using the fine mesh is about $0.3 \%$. The predicted wall skin friction data was extracted from the surface and provided in Fig. 1 with respect to Reynolds number along the chord length. The experimental data obtained by Wieghardt and corrected by Coles [18] is provided for comparison. No significant difference is seen in between the data predicted by all the turbulence model extensions.

\section{Calibration process}

The model extensions described in Chapter III involve five model constants (see Table 1 , assuming that either the extension denoted by PG or V2Pkd are adopted). The traditional practice is to involve an expert-centered manual process for determining the model coefficients. However, the present research involves a range of test cases with partly complex flow behavior, and three model extensions. It was felt that this calibration task cannot be mastered by manual calibration but a more general calibration is sought. We assume that the calibration of uncertain model constants should take into account the known or estimated uncertainties of experimental data. This leads to the approach of Bayesian updates of the uncertain model constants. Due to inherent extremely large number of degree of freedom associated with the probabilistic calibration approach and the significant computational costs of performing unsteady RANS computations, the numerical process employs a number of technical details that are described in the following. 


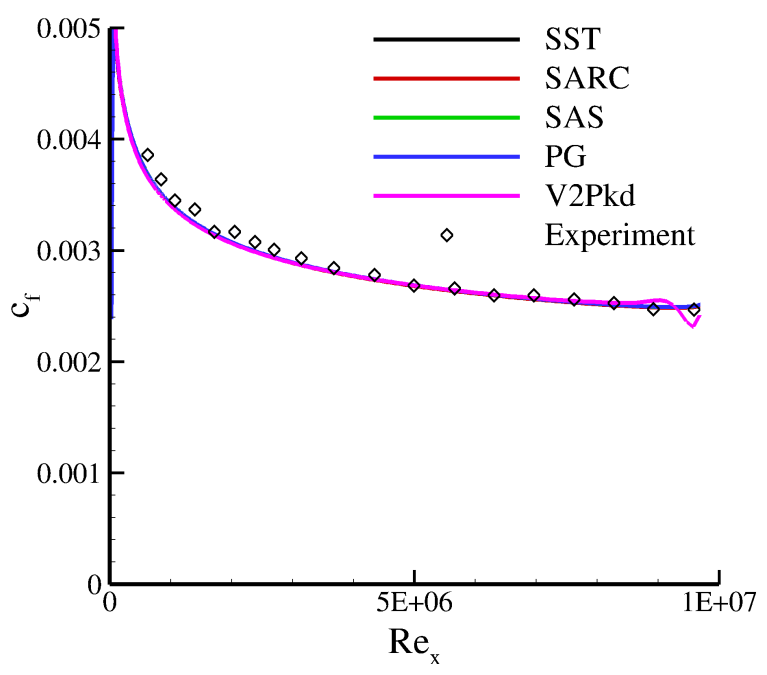

Fig. 1 Skin friction coefficient of ZPGFP

\section{A. Parameter estimation procedure}

Abstractly, the task of parameter estimation is usually formulated as follows. Under consideration is a system with states $\boldsymbol{x} \in \mathbb{R}^{d}$ that are dependent on the uncertain parameters $\boldsymbol{q} \in \mathbb{R}^{N}$, written

$$
\begin{aligned}
\boldsymbol{x}: \mathbb{R}^{d} & \longrightarrow \mathbb{R}^{N} \\
\boldsymbol{q} & \mapsto \boldsymbol{x}(\boldsymbol{q}) .
\end{aligned}
$$

In particular, state is calculable from $\boldsymbol{q}$ via a model. The measurable $\boldsymbol{y}(\boldsymbol{x}) \in \mathbb{R}^{M}$ is a quantity that depends on the system's states. A measurement $\boldsymbol{y}_{m} \in \mathbb{R}^{M}$ is an instance of the measurable that was actually measured, and is usually modeled as the sum of the true measurable and an instance of an additive mean-free noise with distribution $\pi_{\epsilon}$.

The posterior distribution $\pi_{\boldsymbol{q} \mid \boldsymbol{y}_{m}}$ of the parameter $\boldsymbol{q}$ conditioned on our measured response $\boldsymbol{y}_{m}$ can be expressed with the help of the Bayes' formula. The probability distribution of this updated parameter $\overline{\boldsymbol{q}}$ then reads

$$
\pi_{\overline{\boldsymbol{q}}}=\pi_{\boldsymbol{q} \mid \boldsymbol{y}_{m}}(\boldsymbol{q})=\frac{\pi_{\boldsymbol{y}_{m} \mid \boldsymbol{q}}(\boldsymbol{q}) \pi_{\boldsymbol{q}}(\boldsymbol{q})}{\int \pi_{\boldsymbol{y}_{m} \mid \boldsymbol{q}}(\boldsymbol{q}) \pi_{\boldsymbol{q}}(\boldsymbol{q})} .
$$

Here $\pi_{\boldsymbol{q}}$ is the prior distribution of the parameter, our initial guess based on professional expertise amd $\pi_{y_{m} \mid q}$ is the likelihood, the probability of measuring $\boldsymbol{y}_{m}$ given a certain value of the parameters $\boldsymbol{q}$. The likelihood is expressed from the distribution of the measurement noise,

$$
\pi_{\boldsymbol{y}_{m} \mid \boldsymbol{q}}(\boldsymbol{q})=\pi_{\epsilon}\left(\boldsymbol{y}_{m}-\boldsymbol{y}(\boldsymbol{q})\right) .
$$

A one point estimate of the parameter can be given by the most probable value given the measurement

$$
\boldsymbol{q}_{\mathrm{MAP}}=\arg \max _{\boldsymbol{q}} \pi_{\boldsymbol{q} \mid \boldsymbol{y}_{m}}(\boldsymbol{q}),
$$

the so-called maximum a-posteriori (MAP) estimate.

Here, the states are the flow solver's (DLR-TAU) solutions, depending on the $N=5$ parameters of the extended turbulence model as listed in table 1 , and the measurables are the flow field responses such as velocities, Reynolds stresses and surface pressure measured on a spatial grid. Wind tunnel measurements provide the $\boldsymbol{y}_{m}$. Of course the choice of the measurables and their position are geometry-dependent.

\section{B. Bayesian Update}

A random parameter remains a random variable, no matter how well it is estimated using measurements. Giving a point estimate about it hides this fact. Instead it is desireable to estimate as many properties of the posterior random 
variable as possible. Ideally our aim is to get the whole posterior distribution. This way we can see how uncertain our estimate is, and whether there are other values of the parameter giving similar fitting properties of the measurable. Unfortunately, in a general case the Bayesian posterior can not be given in a closed form.

One way to go is to use functional descriptions for all the random variables, the parameter $\boldsymbol{q}$, the measurable $\boldsymbol{y}$ and the additive noise $\epsilon$ and then to try to write the updated random variable $\overline{\boldsymbol{q}}$ with the help of the formers. To describe arbitrary random variables in a discretizeable way, one usually maps a basic random variable that is distributed w. r. t. a very basic distribution, e.g. $\mathcal{N}(0,1), U(0,1)$. The probability of any event in the image is then the probability of all its preimages. The basic random variable is often called the germ. All random variables then are given as a function of the germs. The mappings are approximated by polynomials, reasonably in a basis orthogonal w.r.t. the expectation. This way of approximation of multi-valued random variables is called the generalized polynomial chaos [19].

We still have the task to express somehow the posterior. One could write the updated parameter with the help of a minimum mean squared estimator, e.g. the linear estimator of the Kalman filter which would give us a functional approximation of the updated parameter too. Unfortunately, the Kalman filter is a linear estimator and works well only if the map from the measurable to the updated parameter is linear or mildly nonlinear, which is the case only if (13) is (nearly) linear.

When calculating the gPC coefficients of $\boldsymbol{q}(\omega) \mapsto \boldsymbol{y}$ as presented in Section IV.C. it turns out that this mapping is considerably nonlinear. Thus, the spectral Kalman Filter turned out to be unsuitable for this problem.

The Metropolis-Hastings algorithm, a Markov Chain Monte Carlo Method (MCMC), delivers samples distributed according to the exact Bayesian posterior [20]. The statistics of those samples then provides estimators for the posterior distributions moments, including mean and variance. This algorithm can be regarded to be the working horse for nonlinear parameter estimation problems.

\section{Building a surrogate model}

Doing the random walk of the MCMC procedure requires evaluation of the likelihood at each step. A new step means a new value of the parameter $\boldsymbol{q}$ at which $\boldsymbol{y}(\boldsymbol{q})$ has to be computed for the likelihood with the computationally expensive simulation model. Instead, it is highly advantageous to find a surrogate model for the forward problem (13) in order to circumvent costly evaluations of the DLR-TAU CFD code during sampling.

To choose a suitable surrogate model we tested different meta models [21] for the specific case of the Backward Facing Step (BFS, see Section V.A. The responses $\boldsymbol{y}(\boldsymbol{q})$ here highlighted were the velocity field $v_{x}$ and the Reynolds stresses $\operatorname{Re}_{13}$ along the wall normal direction at four different cross sections of the BFS channel, and the pressure $\left(c_{p}\right)$ and skin friction $\left(c_{f}\right)$ coefficients along the BFS channel. Different sampling techniques were used to generate values of the turbulence model coefficients such as Quasi Monte Carlo based on the Halton sequence, Latin hypercube sampling with random centers and Smolyak grid with different stages. These samples were generated from a 'non-informative' prior distribution of the turbulence model coefficients, a uniform distribution in between the bounds (chosen from literatures and experience gathered during model development) given in Table 1

f The responses have been computed by the CFD simulation for all generated coefficient values. Keeping some

\begin{tabular}{cccccc} 
Phase 1 & PG & SARC_C1 & SARC_C2 & SARC_C3 & SAS \\
\hline \hline left boundary & 0 & 0 & 0 & 0 & 0 \\
right boundary & 0.8 & 1.5 & 10 & 1.5 & 7
\end{tabular}

\section{Table 1 Initial intervals of model coefficients}

samples for testing we cross-validated different surrogate models using $L_{1}, L_{2}$, and $L_{\infty}$ errors. The meta model used were

1) GPC (generalized polynomial chaos) multivariate orthogonal polynomials of maximum total degree three. We used the Legendre polynomials which are orthogonal w.r.t. the given uniform prior distribution.

2) NRBF Normalized radial basis functions with Gaussian kernel with varying parameter $r$, i.e. $g(d)=\exp \left(-(d / r)^{2}\right)$ and $d=\left\|x-x_{i}\right\|$. Centers have been chosen to correspond to the nodes of the training data.

\footnotetext{
*After phase 1, PG model was replaced by V2Pkd model
} 
To generate the surrogate model representations from the training data, the coefficients of the functional approximation were computed by interpolation, regression and projection. Chross-check results of the different approximation methods using different parameters and using different data sets for training and validation data have been compared and evaluated. From this validation we concluded to use the gPC in the further analysis. Though in this initial testing the first degree gPC surrogate had the mimimum error, in later analysis we computed more sample points and repeated the validation process only for the different degree gPC models by regression. This was necessary because the regression with the small number of sample points were underdetermined. With more sample points higher degree gPC models improved, and so the expansion of total-degree 3 was chosen.

\section{Maximum a posteriori estimates}

From the distributed samples one chooses the the center of the histogram's interval with the highest density as estimator $\hat{\boldsymbol{q}}$. This approximates the point with the highest a pasteriori density. These are taken as the coefficients of the calibrated turbulence model.

In this paper, we apply the procedure separately to all the generic test cases described in Section $\mathrm{V}$ separately and to measurements joint together from two groups of two resp. 3 of the generic test cases. The first set is a combined update involving the Backward Facing Step, axisymmetric transonic Bump and Model 53 at $\alpha=20 \mathrm{deg}$. Second set, which involves the flow with vortex breakdown, encompasses the two higher angles of attack with Model 53 $(\alpha=25 \mathrm{deg}$ and $28 \mathrm{deg}$ ). A sample a priori and a posteriori probability distribution of the parameters and its cross dependencies are shown in Fig. 2. The calibrated coefficients are given in the following table 2, using which the
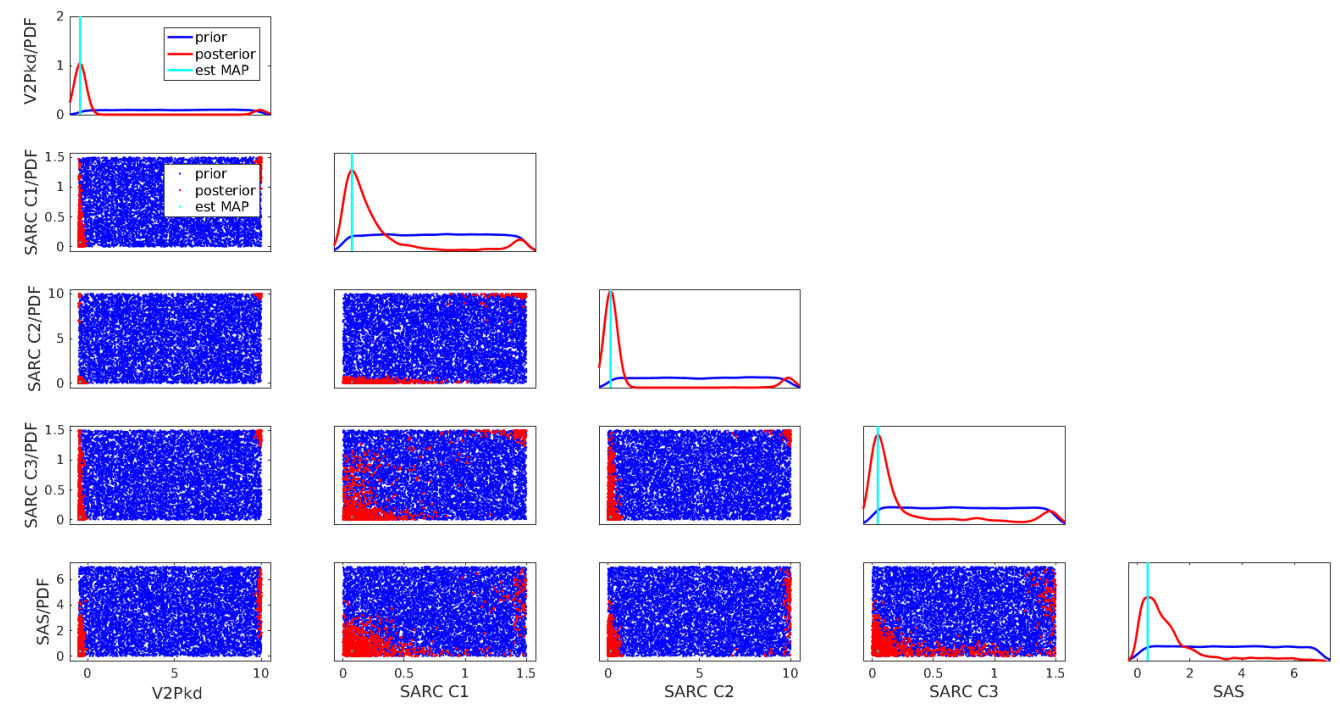

Fig. 2 Combined Bayesian update of Model 53 delta wing at angle of attack 25 deg and 28 deg

simulations are performed and illustrated in the results section $\mathrm{V}$

\begin{tabular}{cccccc} 
& V2Pkd & SARC_C1 & SARC_C2 & SARC_C3 & SAS \\
\hline \hline Set 1 & 1.82 & 1.46 & 3.29 & 0.01 & 2.18 \\
\hline \multicolumn{7}{c}{ Set 2} & -0.42 & 0.08 & 0.15 & 0.05 & 0.32
\end{tabular}

Table 2 Calibrated parameters of the turbulence model extensions 


\section{Generic Test Cases}

The calibrations are performed on different flow problems involving two-dimensional (2D) and three-dimensional (3D) test cases. The predominant test and application cases comprise low aspect ratios wings. Therefore, two different types of delta wings are being studied associated with complex vortical flows. A particular difficulty is related to the leading edge shape of delta wings as the separation is not fixed in the case of a delta wing with round leading edge. Moreover vortex interactions take place, which increases the complexity furthermore. A well known problem is the challenging task to effectively predict the exact onset of separation using a turbulence model and the chracteristics of the vortices involved. A first phase of the calibration was done employing the Backward Facing Step, axisymmetric transonic Bump and Model 53 delta wing at an angle of attack of $20 \mathrm{deg}$. Calibrations were conducted successively, including one test case after the other. However the objective is to perform combined calibration and therefore only the final result involving the combined update results are shown in this paper. The flow computations performed with set 1 of model constants also involved higher angles of attack where vortex breakdown occurred. Here, it became apparent that set 1 resulted in a too early vortex breakdown. Therefore, a second calibration devoted to predict vortex breakdown was performed that employs the angles $\alpha=25 \mathrm{deg}$ and $\alpha=28 \mathrm{deg}$.

\section{A. Backward Facing Step}

Backward Facing Step (BFS) is a standard test case involving flow separation at a fixed point. It is relatively easy to measure and model and the prediction of point of flow reattachment along with flow properties downstream is of primary importance. The simulation uses flow information extracted from an initial channel computation as inflow condition, thereby reducing the computational effort. The computational grid available in the Nasa turbulence homepage was used [22]. The Mach number is 0.2 , step height is $0.0127 \mathrm{~m}$ and the Reynolds number based on the step height is 38000. Unsteady RANS simulations were performed with a low Mach number preconditioning. Second order central scheme was used for spatial and backward Euler was used for temporal discretization. Experimental data for reference is obtained from Driver and Seegmiller [22]. For the calibration purpose, 300 samples of parameters were used to provide a CFD data of the model, which were then used in UQ suite to build the surrogate model and perform the Bayesian Update. While the combined update involves bump and Model 53( $\alpha=20 \mathrm{deg})$ test case as provided in the following sections, a stand alone update performed only with BFS is also included here to serve as an initial example of calibration.

Figure 3 shows the surface pressure coefficient predicted by the updates. Based on the sensitivity analysis performed with the individual extensions, the pressure gradient extension augments to a slightly larger suction peak. The prediction of wall shear stress coefficient is as expected (Fig. 3 $\mathrm{p}$ ), in which the SAS extension, by adding the sink term to the $\omega$-equation, reduces dissipation and increases turbulence thereby causing earlier reattchment than other models. The irrotational strain model influences the update to predict a later reattachment point, as this part sensitises the model to pressure gradients. The SARC model has the least influence on the original model out of the three extensions. The velocity and Reynolds stress profiles in the boundary layer are shown in the Fig. 3 at two downstream locations. It can be seen that the Reynolds stress is significantly increased by the updates at one step height downstream of the flow direction but the magnitude of the reverse flow is slightly larger than that of the SST model and that of the experiment. As the flow progresses further downstream, the velocity profiles of the updates are improved over the original model although the Reynolds stress components are larger than the original model and experiment in the corresponding section. This tendency has been observed by other classical turbulence models as well.

\section{B. Axisymmetric Transonic Bump}

The choice of the transonic axisymmetric bump as a test case is justified by the need of ensuring the model performance for predicting shock/boundary layer interaction with flow separation and reattachment. Flow conditions are $\mathrm{Ma}=0.875$ at a unit Reynolds number of $\mathrm{Re} / \mathrm{m}=13.6 \times 10^{6}$. A strong compression shock occurs and, combined with subsonic flow diffusion towards the bump trailing edge, leads the flow to separation. The flow starts recovering downstream of the bump trailing edge, where it reattaches. The computational grid available in the Nasa turbulence homepage was used [22]. The Mach number is 0.875, the Reynolds number based on chord length is 2.763 million. Steady RANS simulations were performed along with a second order upwind scheme for spatial and backward Euler method was used for temporal discretization. Experimental data for reference is obtained from Bachalo and Johnson [22]. For the calibration purpose, 300 samples of parameters were used to provide the CFD data of the model, which were then used in UQ suite to build the surrogate model and perform the Bayesian Update. The combined update involves BFS and Model 53( $\alpha=20 \mathrm{deg})$ test case as provided in the previous and following sections and for reference 
purpose, stand alone update performed only with bump is also included.

The surface pressure coefficient along the bump is shown in Fig. 4a. It has to be noted that the original SST model was calibrated using the transonic bump case, thereby delivering quite a good agreement with the experiment. Calibrated results of the extended model predict the shock location slightly downstream than that of the SST model and experiment. The pressure increase is relatively steep behind the shock. From sensititvity calculations not shown here, it was observed that the SAS extension is effective at shock and it contributes to the disagreement of the pressure coefficient. Another contribtuting factor can be seen from the boundary layer profiles. The surrogate model provides equal weight to all the available experimental data and since a large set of data points are available for velocity and Reynolds stress profiles, a strong weight is given to improving the boundary layer profiles. At $\mathrm{x} / \mathrm{c}=0.688$ where the shock takes place, the extended model improves the velocity profile significantly as can be seen in the Fig. $4 \mathrm{~b}$. At $\mathrm{x} / \mathrm{c}=1.125$, the original SST model still predicts a reverse flow, whereas the extended model has improved the result tending towards an attached flow (4p). Corresponding improvement is also seen by the increased Reynolds stress magnitude and agreement of the profile shape with the experiment at the same location (4d). Further downstream at $\mathrm{x} / \mathrm{c}=1.375$, the improvement in the flow recovery region can be seen in the velocity profile (44). Reynolds stress profiles in the region are similar with each other between the original and extended model.

\section{Model 53}

Model 53 (Fig. 5 \& 6) is a relatively sharp leading edge delta wing (leading edge radius at the root chord is 1.22 $\mathrm{mm}$ ) with a sweep angle of $53 \mathrm{deg}$ and along with a leading edge slat deployed at $20 \mathrm{deg}$. The root chord is $0.75 \mathrm{~m}$ and the wing is twisted at an angle of $-4 \mathrm{deg}$ at the tip. Measurements of pressure distributions were provided by Technical University of Munich in its low speed wind tunnel A [23]. The flow conditions are a Mach number of 0.2 and Reynolds number based on the mean aerodynamic chord $(0.51 \mathrm{~m})$ is 1.7 million. Due to the small radius of the leading edge, the onset of the primary vortex takes place in the proximity of the apex. Apart from the primary leading edge vortex, a second leading edge vortex develops at the beginning of the slat. Based on the flow conditions and angle of attack, onset of a third vortex is also observed downstream, near the trailing edge section. Vortex interactions take place between the vortices originating from the delta wing apex section as well as from the slat, which adds up to the inherent complexity of the vortical flows. Breakdown of the vortices occur due to the presence of adverse pressure gradient towards the trailing edge. Moreover, as the angle of attack increases the vortex breakdown location move upstream. This physical phenomenon is highly unsteady which could have adverse effects on the flight control parameters due to the abrupt change of the pressure distribution on the suction side of the wing. Therefore it is important to accurately predict the location and mode of vortex breakdown. Three different angles of attack, namely $20 \mathrm{deg}, 25 \mathrm{deg}$ and 28 deg were computed. The size of the numerical grid was around 8.85 million and unsteady RANS simulations were performed with a low Mach number preconditioning. Second order Central scheme was used for spatial discretization and backward Euler was used for temporal discretization.

\section{Calibration Set 1: $\alpha=20$ deg}

During the first phase of calibration, 100 samples of parameters were used to obtain the CFD data for the Model 53 test case at $\alpha=20 \mathrm{deg}$, which were then used in the UQ suite to build the surrogate model and perform the Bayesian Update. The combined update involves BFS and bump test case as provided in the previous sections. Distributions of spanwise surface pressure coefficient at the suction side along 16 sections of the wing comprise the flow state which was used during the calibration process. A number of sections at the pressure side were used to make sure that the extended model would not adversely affect the pressure side. The calibrated results are shown in Fig. 7 Note the suction peak of the apex vortex onset around apex region is somewhat larger for the experiment that that of the original SST model and the extended model. At around $21 \%$ of the root chord length, the footprint of the apex vortex from the experiment matches well with both models and also the suction pressure coefficient is improved by the extended model. The emergence of a slat vortex at the outboard part of the wing can be seen at this section and it is reaffirmed around $26 \%$ of the chord length. The dissipation of the first vortex is captured well by the extended model around $30 \%$ to $40 \%$ of the root chord length, and the improvements versus the original model become apparent. The tendency continues as the flow progresses towards the trailing edge of the wing. However, the occurence of the third vortex is not captured by both models. 


\section{Calibration Set 2: $\alpha=25 \mathrm{deg}$ and $\alpha=28 \mathrm{deg}$}

The second phase of calibration employed the angles of attack of $25 \mathrm{deg}$ and $28 \mathrm{deg}$ and it was noted that the extended model is sensitive to the parameters involved. Moreover, it became apparent that the V2Pkd model was also effective in the negative parameter region. Therefore, along with the existing parameter interval used in phase 1, ten additional parameter samples were added to the existing 100 samples. Therefore, in total 110 simulations were performed for the two higher angles of attacks. For the angle of attack of $25 \mathrm{deg}$, the spanwise surface pressure coefficients are shown in Fig. 8 . It can be seen that the original SST model displays a quite good agreement with the experimental data. The onset and footprint of the apex vortex is well captured by both models as it can be seen around $20 \%$ of the root chord length. However, a lower suction peak of the slat vortex is seen outboard near the wing tip, by both indicated models. Around 30\% to $40 \%$ of the root chord length, the characterstics of the second vortex are improved by the calibrated model near to the wing tip. The vortex breakdown occurs around the middle part of the wing. The dissipation of the vortices towards the trailing edge predicted by the numerical models is in good agreement with the experiment, towards the trailing edge. The pressure coefficient is slightly over predicted by the extended model.

Figure 9 shows the calibration results of the Model 53 test case at 28 deg angle of attack. The occurence of vortex breakdown takes place much more upstream for this high angle of attack. As for the previous case, the standard SST model exhibits a good agreement with the experiment. Near to the apex, at around $4 \%$ of the root chord length, the pressure distribution is slightly improved by the extended model. However, around $10 \%$ the standard model has a slightly better agreement, as a slight increase in pressure is predicted by the extended model. Both of the numerical models predict a somewhat larger pressure coefficient further downstream, whereas a very good agreement is found near the trailing edge of the wing around $80 \%$ of the root chord length. It can be seen that the flow characteristics at an anlge of attack associated with vortex breakdown is predicted well by both models.

\section{Validation using Model 56}

Model 56 is a variant of the Model 53 delta wing, the difference being the sweep angle, which is 56 deg for the Model 56 test case. The test conditions remains the same as for the Model 53. The dependency of the vortex characteristics are influenced by the sweep angle, such as the change in the magnitude and the footprint of the vortex on the suction side. The objective of using the Model 56 as a validation case is to determine the usefulness of the calibration for subsequent design applications. Since the calibration process does not include an a priori information about the flow characteristics of this test case, it is a suitable case for validation. Throughout the course of this project, an alternative model calibration approach based on deterministic optimization of model coefficients was studied by Airbus Defence and Space and Technical University of Munich. The results from the standard Spalart Alamaras (SA) turbulence model and an optimized SA-model are added as a reference. [24]

\section{Validation of Set 1: $\alpha=20 \mathrm{deg}$}

The validation results are shown in Fig. 10 Around 13\% of the root chord, near to the apex region of the delta wing, the strength of the vortex core is suppressed by both the SST and extended model, with SST in better agreement. At $40 \%$ the disspiation of the apex vortex is well predicted by both the models along with good agreements towards the footprint and magnitude of the slat vortex. Further downstream, the magnitude of the slat vortex is reduced by the numerical models. As it was observed with the calibration cases, the occurence of the third vortex around $60 \%$ of the chord is not predicted. This leads to the difference in the pressure coefficient towards the wing tip for the trailing edge region.

\section{Validation of Set 2: $\alpha=25 \mathrm{deg}$ and $\alpha=28 \mathrm{deg}$}

Figure 11 shows the Model 56 results at $\alpha=25 \mathrm{deg}$. Improvements in the pressure distribution for the apex region can be seen by applying the extended model. Around 30\% to $40 \%$ of the chord length, the apex vortex is well predicted, whereas the slat vortex is predicted slightly inboard by the numerical models. Also, the magnitude of the suction peaks are improved by the extended model. The pressure coefficient distribution is improved also in the middle region of the wing and both models are in good agreement towards the trailing edge of the wing. The validation results of the Model 56 test case at $\alpha=28 \mathrm{deg}$ are shown in Fig. 12. Around 30\% of the wing chord length, the apex vortex is predicted well by both models whereas the magnitude of the slat vortex near to the trailing edge is improved by the extended model. However, the footprint of the second vortex obtained by the numerical models is slightly located outboard in 
comparison to the experiment. The pressure coefficient is improved by the extension around the middle part of the wing, especially at the wing tip region. Further downstream, the data of the numerical models are well in agreement with the experimental data for the trailing edge region. As it was the case with the Model 53, it can be seen that the flow chracteristics at an agle of attack provoking vortex breakdown are predicted well.

\section{Summary}

Extensions to the standard Menter-SST eddy viscosity turbulence model were studied, developed and implemented in the DLR-TAU CFD flow solver. The numerical simulations are coupled with a internally developed Uncertainty Quantification suite. Calibrations are performed with standard test cases involving backward facing step, axisymmetric transonic bump and Model 53 delta wing. Successive Bayesian Updates were performed and the extended model was calibrated in two phases. Improvements can be seen by the extended turbulence model over the standard model. A delta wing test case uninvolved in the calibration process was then used for the extended model validation. Improvements were seen in all the involved test cases. Therefore, the work highlights the potentials of physical based turbulence model extensions and calibration through Bayesian Updates. Further studies will be undertaken using a refined region of interest to establish a single set of parameters for the extensions involving delta wing test cases, which could then be applied to vortical flows and vortex breakdown cases.

\section{Acknowledgments}

The authors gratefully acknowledge LuFo V - "Vitual Aircraft Model-Calibration" (LUFOV2-790-024/FKZ:20A1504A) for funding this research project. The turbulence modeling group of the VitAM project is acknowledged for the numerous discussions, knowledge and data transfer. DLR is acknowledged for the TAU code and informative discussions. NASA turbulence resource homepage is acknowledged for the numerical meshes and experimental data. The computations shown in this paper were performed using the HLRN (Norddeutscher Verbund für Hoch- und Höchstleistungsrechnen), which was essential for the cases requiring large resources. Development of the UQ suite was supported through “2018-1.2.1-NKP-00008 Exploring the Mathematical Foundations of Artificial Intelligence”.

\section{References}

[1] Menter, F. R., "Sensitizing of the SST Turbulence Model to Rotation and Curvature by Applying the Spalart-Shur Correction." Journal of Turbomachinery, Vol. 131, 2009.

[2] Polhamus, E. C., "Prediction of Vortex-Lift Characteristics by a Leading-Edge Suction Analogy," Journal of Aircraft, Vol. 8, 1971.

[3] Lambourne, N. C., and Bryer, D. W., "The Bursting of Leading-Edge Vortices-Some observations and Discussion of the Phenomenon," Aeronautical Research Council, Reports and Memoranda, , No. 3282, April 1961.

[4] Fritz, W., "Numerical solutions for the VFE-2 configuration on the structured grids at EADS-MAS," Aeronautical Research Council, Reports and Memoranda, , No. ISBN 978-92-837-0073-9, 2009.

[5] Boyden, R. P., "Effects of Leading-Edge Vortex Flow on the Roll Damping of Slender Wings," Journal of Aircraft, , No. 7, July 1971.

[6] Carlson, H. W., and Mack, R. J., "Studies of Leading-Edge Thrust Phenomena," Journal of Aircraft, Vol. 17, No. 12, December 1980.

[7] Hak, M. G., and Blackwelder, R. F., “The Discrete Vortices from a Delta Wing,” AIAA Technical Notes, June 1985.

[8] Menter, F., “Zonal Two Equation k- $\omega$ Turbulence Models for Aerodynamic Flows.” AIAA, Vol. 93-2906, 1993.

[9] Knight, D. D., and Saffman, P. G., "Turbulence Model Predictions for Flows with Significant Mean Streamline Curvature." AIAA 16th Aerospace Sciences Meeting, 1978.

[10] Spalart, P. R., and Shur, M., "On the Sensitization of Turbulence Models to Rotation and Curvature." Aerospace Science and Technology, Vol. 5, 1997, pp. 297-302.

[11] Shur, M. L., Strelets, M. K., and Travin, A. K., “Turbulence Modeling in Rotating and Curved Channels: Assesing the Spalart-Shur Correction.” AIAA Journal, Vol. 38, 2000, pp. 784-732. 
[12] Smirnov, P. E., and Menter, F. R., "Turbulence Modeling in Rotating and Curved Channels: Assesing the Spalart-Shur Correction.” Journal of Turbomachinery, Vol. 131, 2009.

[13] Egorov, Y., and Menter, F., "Development and Application of SST-SAS Turbulence Model in the DESIDER Project." Advances in Hybrid RANS-LES Modelling, Vol. NNFM 97, 2008, pp. 261-270.

[14] Maduta, R., "An Eddy-Resolving Reynolds Stress Model for Unsteady Flow Computations: Development and Application.” Dissertation, Technische Universität Darmstadt, 2014.

[15] Cécora, R.-D., Reynolds-Spannungsmodell der Turbulenz für Anwendungen der Flugzeugaerodinamik, Dissertation, Technische Universität Braunschweig, 2015.

[16] Hanjalic, K., and Launder, B. E., "Sensitizing the Dissipation Equation to Irrotational Strains.” Journal of Fluids Engineering, Vol. 102, 1980, pp. 34-40.

[17] Probst, A., "Reynoldsspannungsmodellierung für das Überziehen in der Flugzeugaerodynamik." Dissertation, Technische Universität Braunschweig, 2013.

[18] Wieghardt, K., and Tilman, W., “On the Turbulent Friction Layer for Rising Pressure,” NACA TM-1314, 1951.

[19] Najm, H. N., "Uncertainty quantification and polynomial chaos techniques in computational fluid dynamics," Annual review of fluid mechanics, Vol. 41, 2009, pp. 35-52.

[20] Rosić, B., Sỳkora, J., Pajonk, O., Kučerová, A., and Matthies, H. G., "Comparison of numerical approaches to Bayesian updating," Computational Methods for Solids and Fluids, Springer, 2016, pp. 427-461.

[21] Friedman, N., and Zander, E., "Vitam Report," Institute of Scientific Computing, TU BS, 2018.

[22] Rumsey, C., "Langley Research Center Turbulence Modeling Resource,", stand 2018. URL https://turbmodels. larc. nasa.gov/

[23] Moioli, M., Reinbold, C., Sorensen, K., and Breitsamter, C., "Investigation of Additively Manufactured Wind Tunnel Models with Integrated Pressure Taps for Vortex Flow Analysis,” MDPI Aerospace, 2019.

[24] Moioli, M., Sorensen, K., and Breitsamter, C., "Turbulence Modeling for Leading-Edge Vortices: an Enhancement based on Experimental Data, Control ID 3229270,” AIAA Scitech, 2020. 


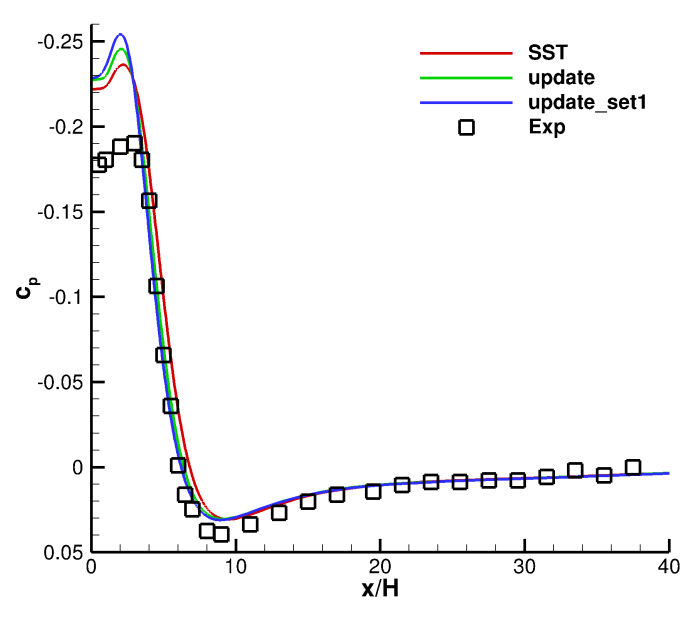

(a) $c_{p}$ coefficient

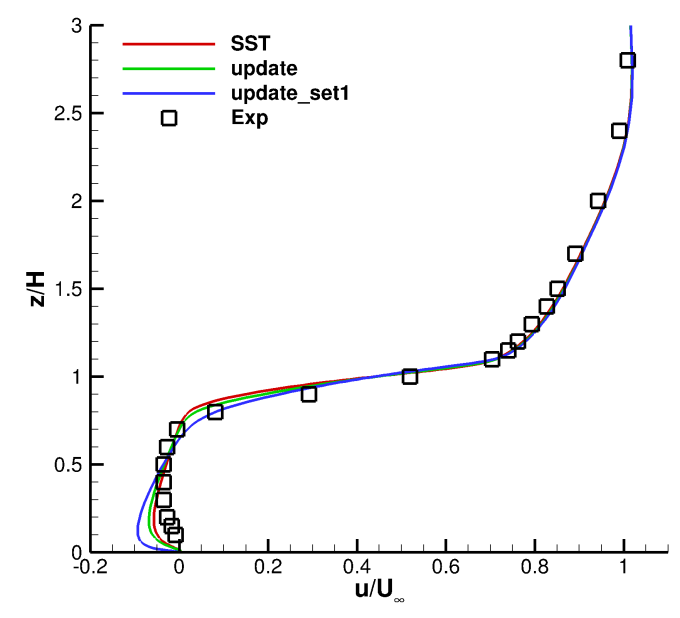

(c) normalized streamwise velocity at $\mathrm{x} / \mathrm{H}=1$

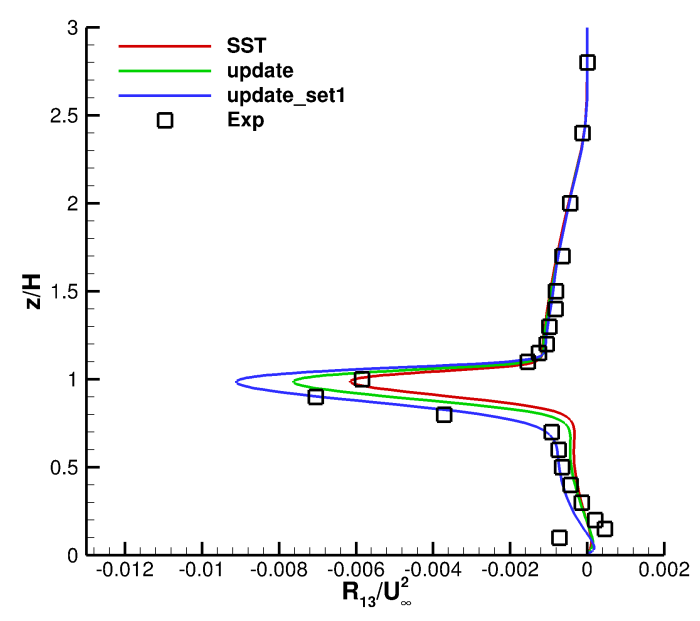

(e) normalized shear stress $\left(R_{13}\right)$ at $\mathbf{x} / \mathbf{H}=\mathbf{1}$

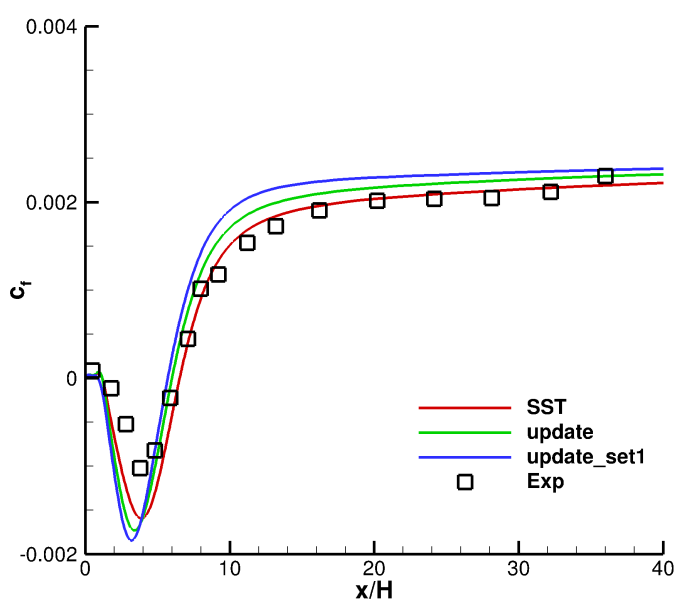

(b) $c_{f}$ coefficient

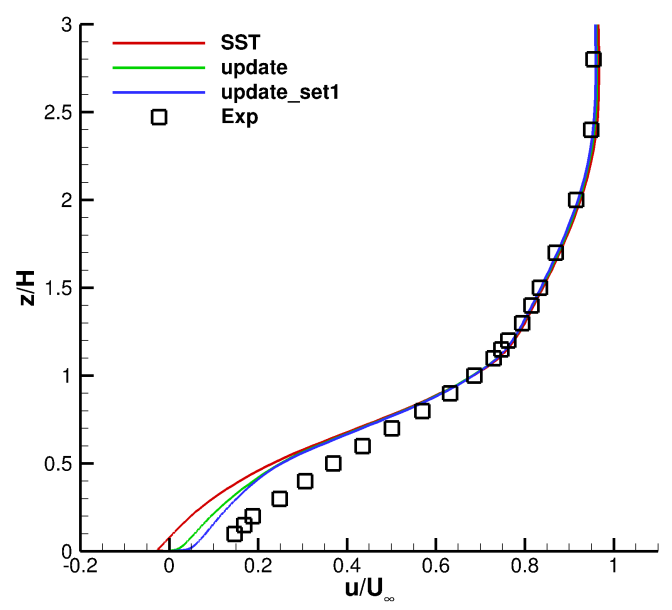

(d) normalized streamwise velocity at $\mathrm{x} / \mathrm{H}=6$

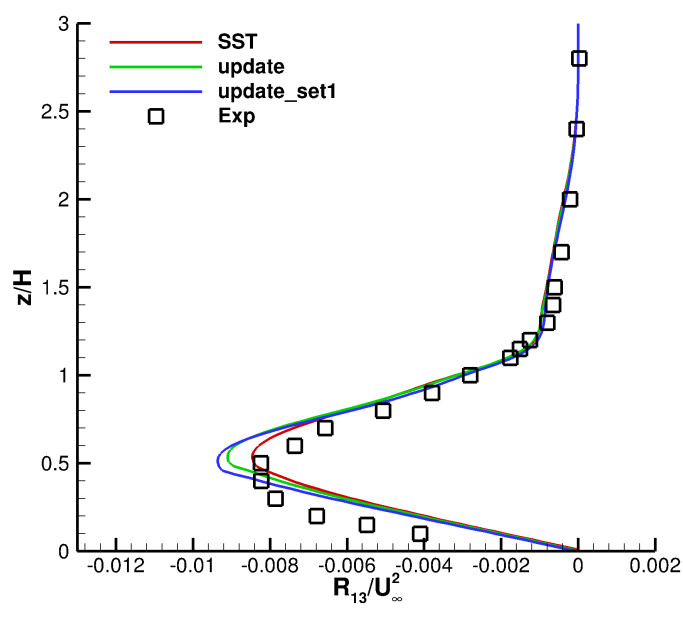

(f) normalized shear stress $\left(R_{13}\right)$ at $\mathbf{x} / \mathbf{H}=6$

Fig. 3 DLR-TAU response for BFS calibration 


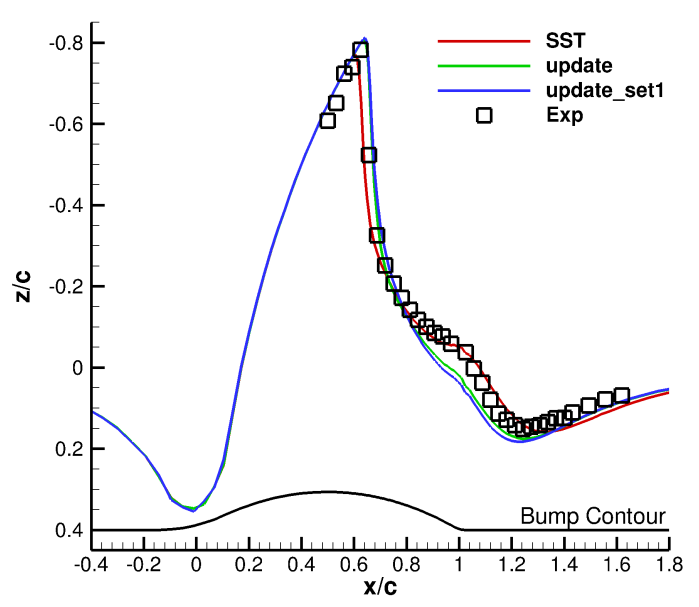

(a) $c_{p}$ coefficient

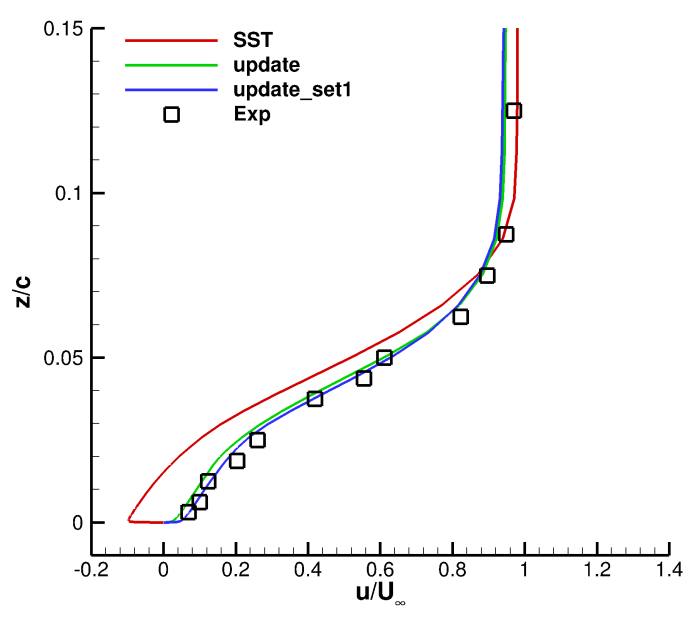

(c) normalized streamwise velocity at $\mathrm{x} / \mathrm{c}=\mathbf{1 . 1 2 5}$

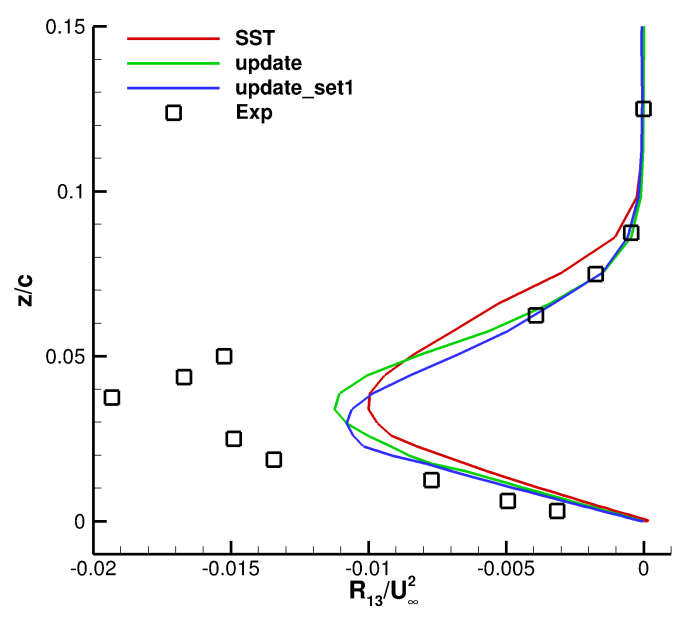

(e) normalized shear stress $\left(R_{13}\right)$ at $\mathbf{x} / \mathbf{c}=\mathbf{1 . 1 2 5}$

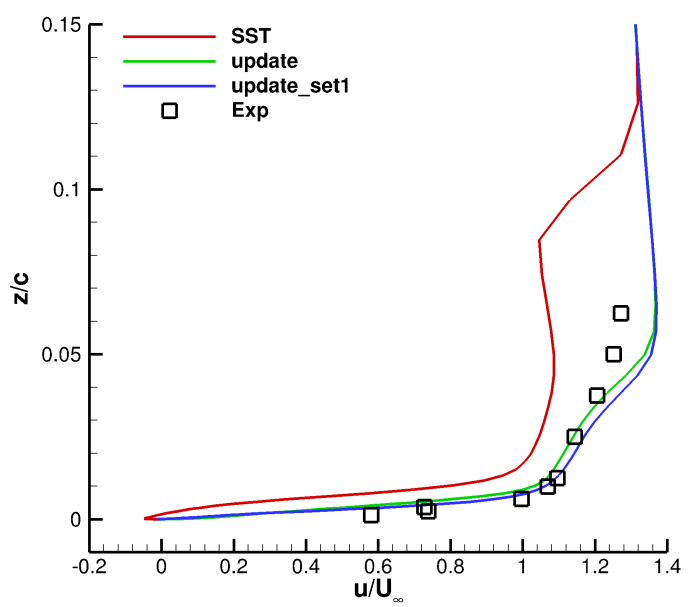

(b) normalized streamwise velocity at $\mathrm{x} / \mathrm{c}=\mathbf{0 . 6 8 8}$

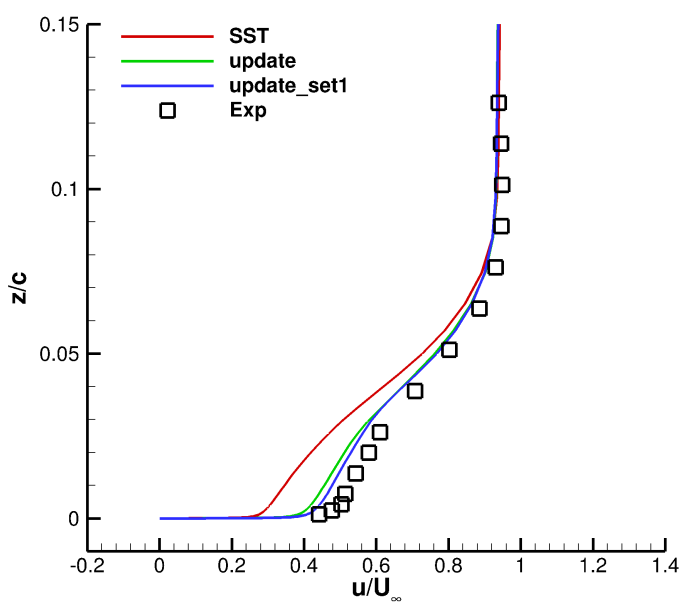

(d) normalized streamwise velocity at $\mathrm{x} / \mathrm{c}=\mathbf{1 . 3 7 5}$

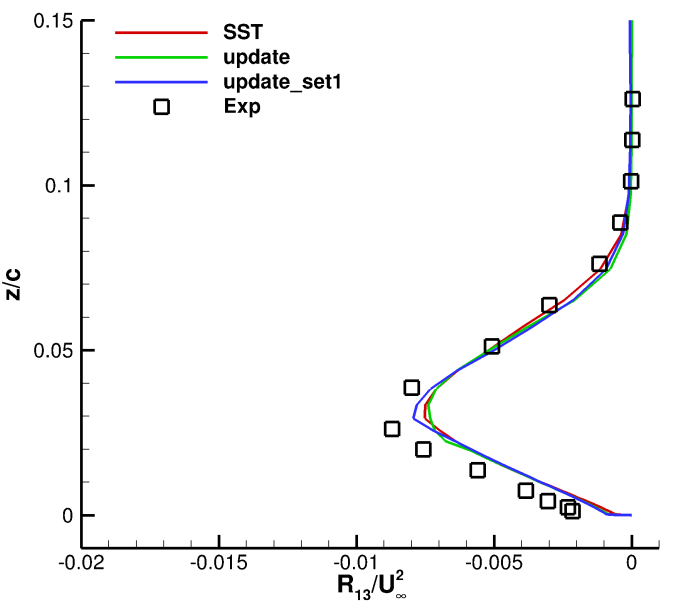

(f) normalized shear stress $\left(R_{13}\right)$ at $\mathbf{x} / \mathbf{c}=1.375$

Fig. 4 DLR-TAU Response for axisymmetric transonic bump calibration 


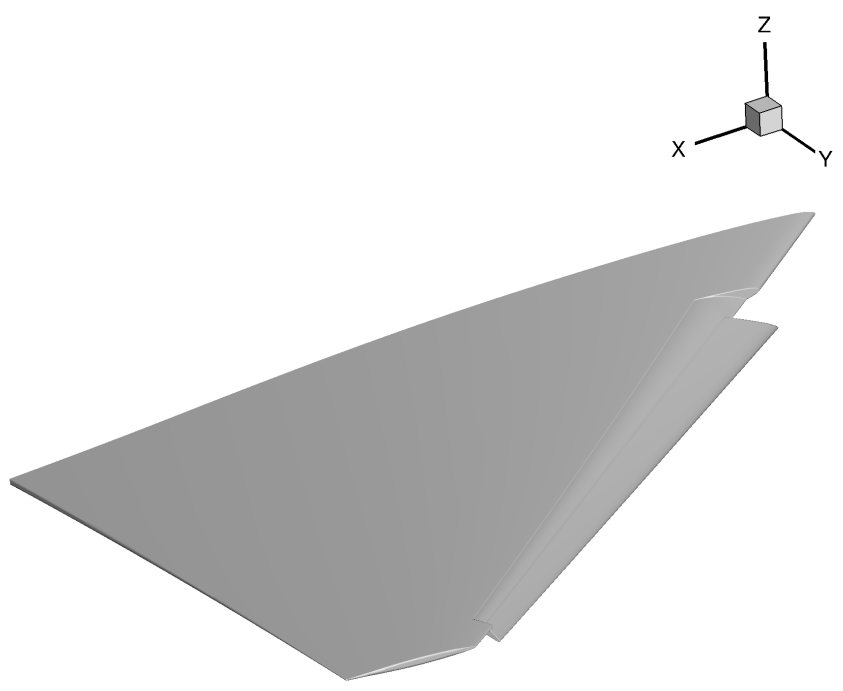

Fig. 5 Model 53 delta wing

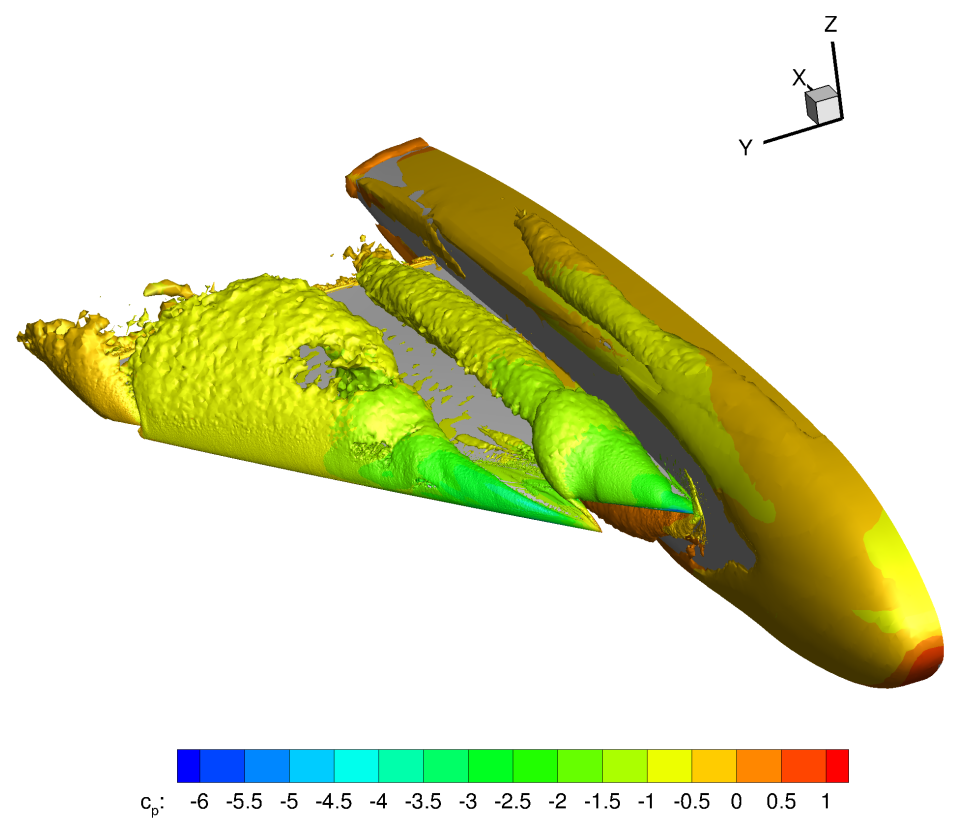

Fig. 6 Illustration of Model 53 with fuselage and peniche; Iso surfaces using non dimensional Q-criterion (0.001) at $\alpha=25 \mathrm{deg}$, SST model 


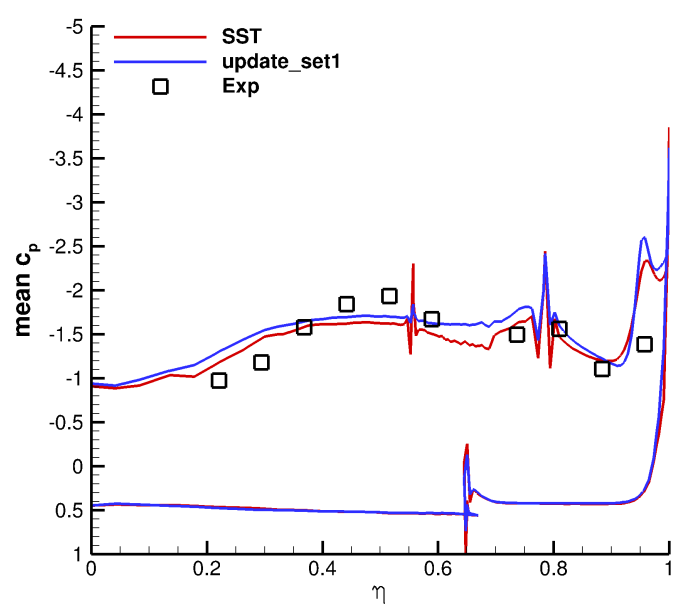

(a) $x / c_{r}=\mathbf{2 1 . 3 3 \%}$

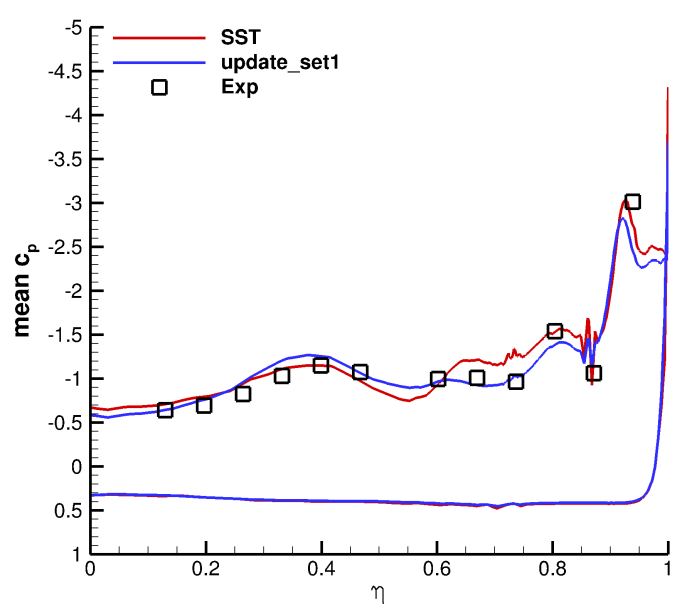

(c) $x / c_{r}=\mathbf{3 2 \%}$

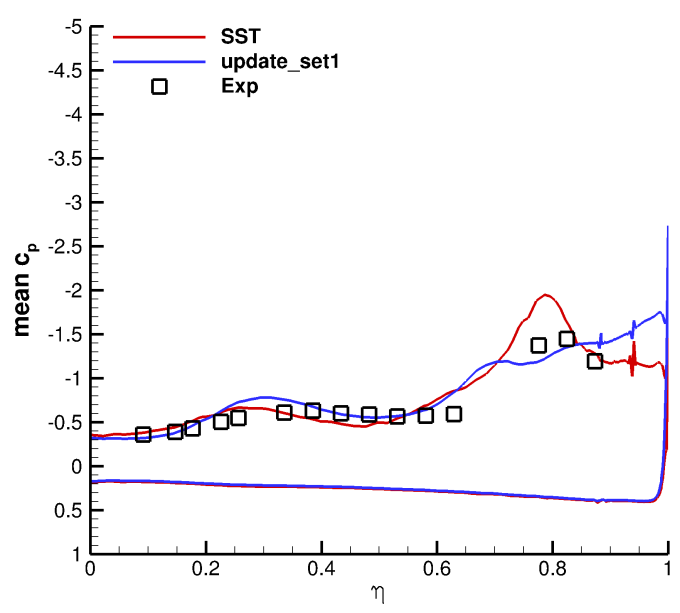

(e) $x / c_{r}=\mathbf{5 6 . 6 6 \%}$

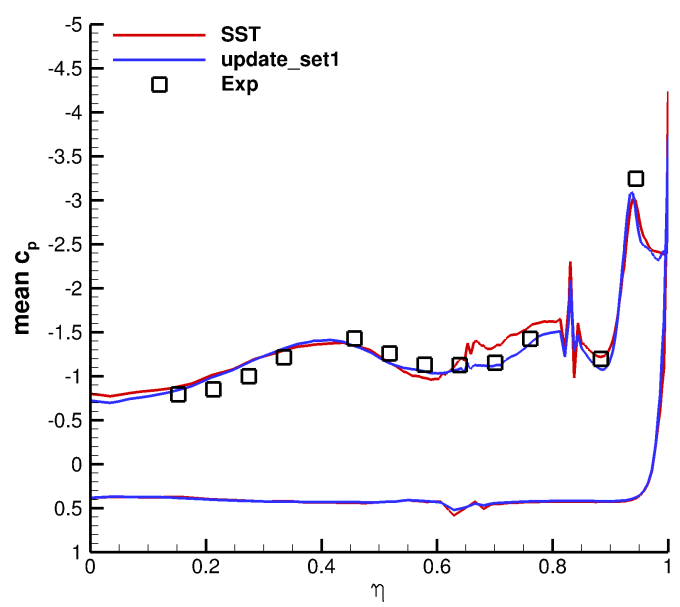

(b) $x / c_{r}=\mathbf{2 6 . 6 6 \%}$

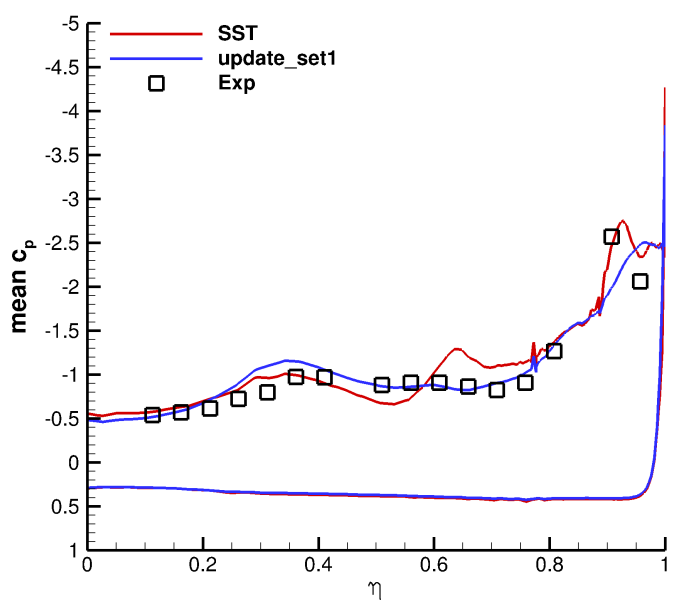

(d) $x / c_{r}=\mathbf{3 7 . 3 3 \%}$

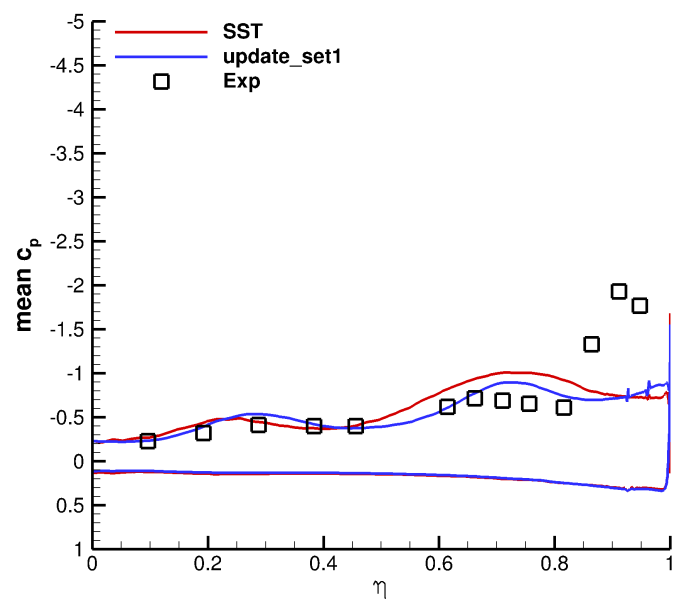

(f) $x / c_{r}=72.66 \%$

Fig. 7 Calibration of spanwise surface pressure coefficient of Model 53 delta wing at $\alpha=20 \mathrm{deg}$ 


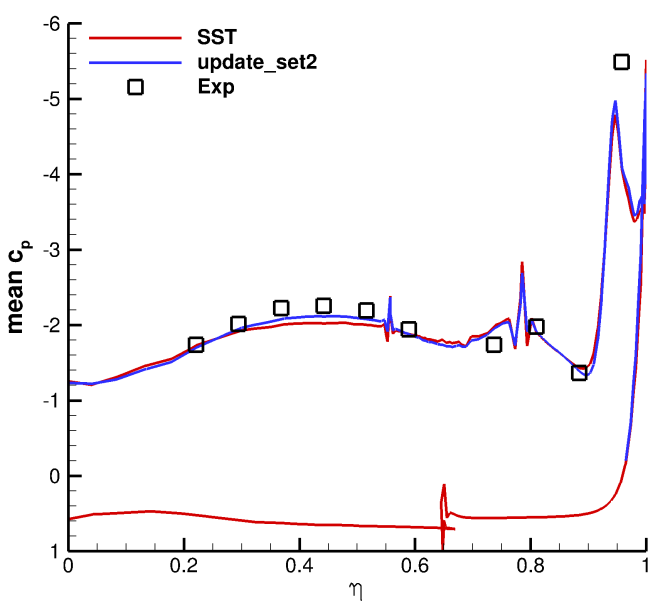

(a) $x / c_{r}=\mathbf{2 1 . 3 3 \%}$

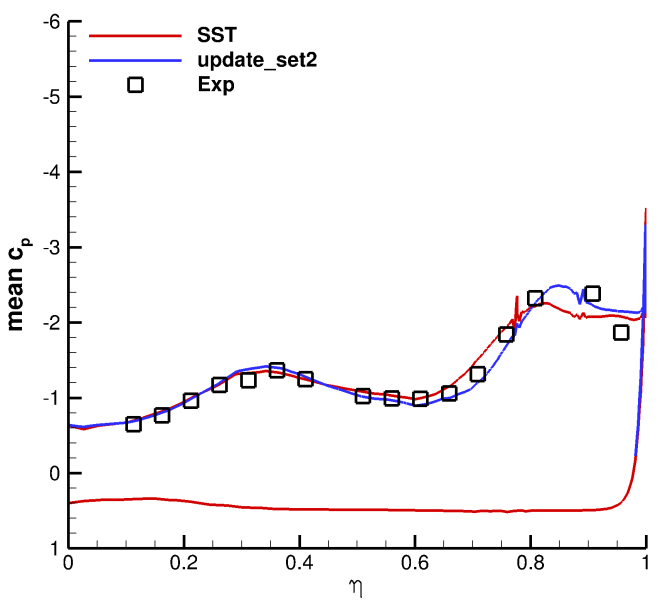

(c) $x / c_{r}=\mathbf{3 7 . 3 3 \%}$

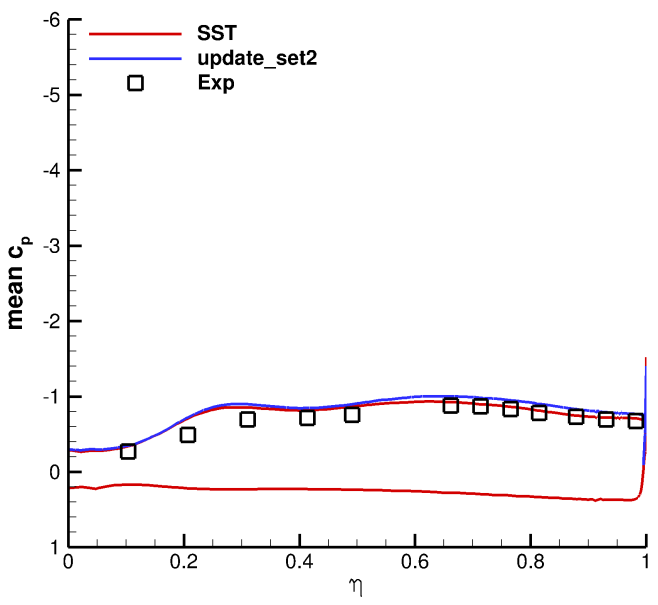

(e) $x / c_{r}=67.33 \%$

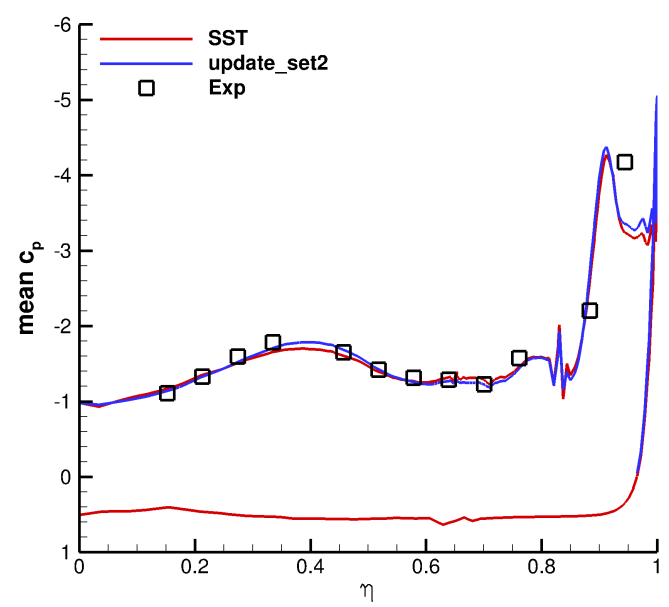

(b) $x / c_{r}=\mathbf{2 6 . 6 6 \%}$

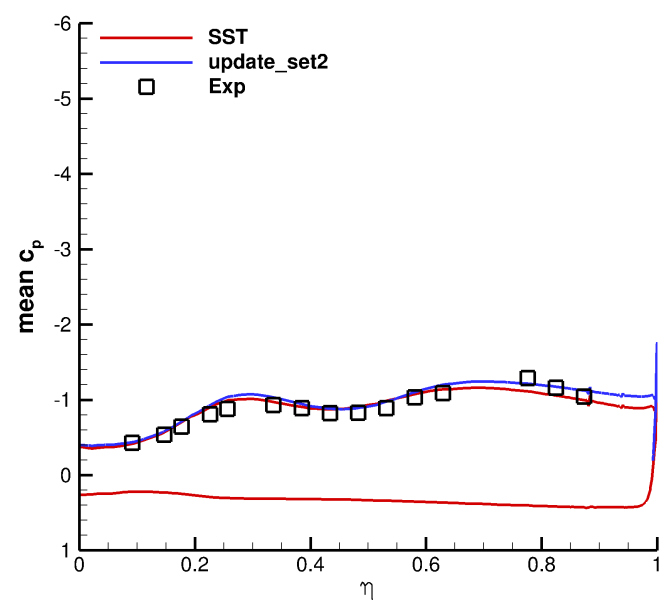

(d) $x / c_{r}=\mathbf{5 6 . 6 6 \%}$

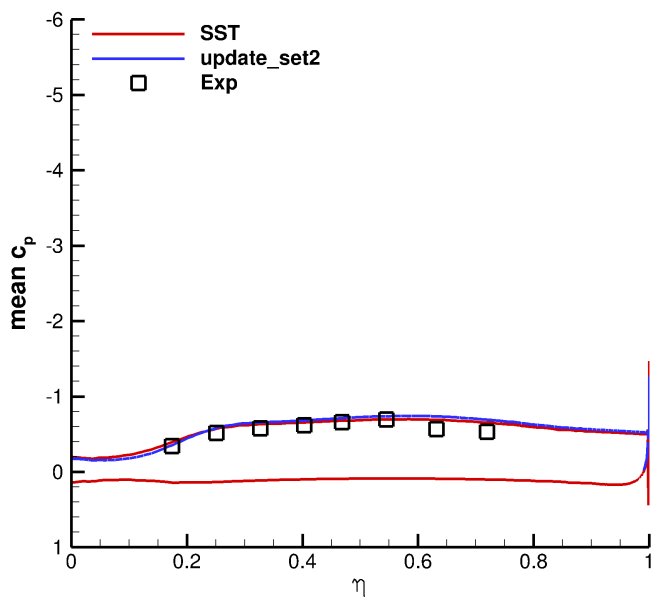

(f) $x / c_{r}=\mathbf{8 2 . 6 6 \%}$

Fig. 8 Calibration of spanwise surface pressure coefficient of Model 53 delta wing at $\alpha=25 \mathrm{deg}$ 


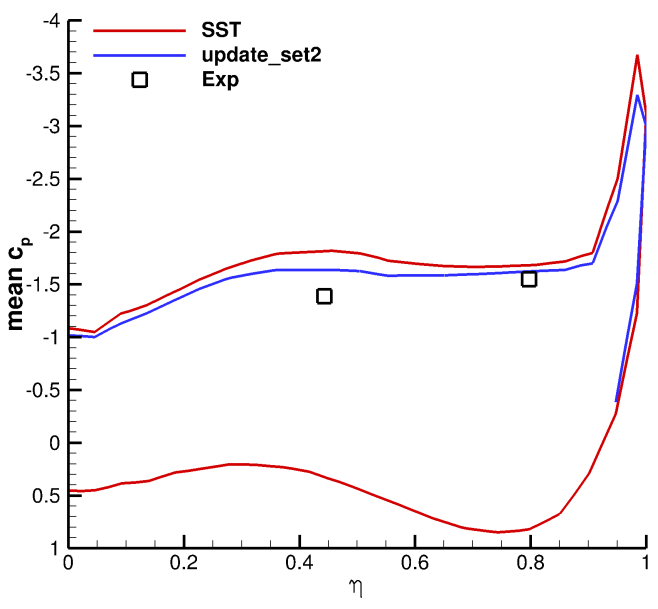

(a) $x / c_{r}=\mathbf{4 \%}$

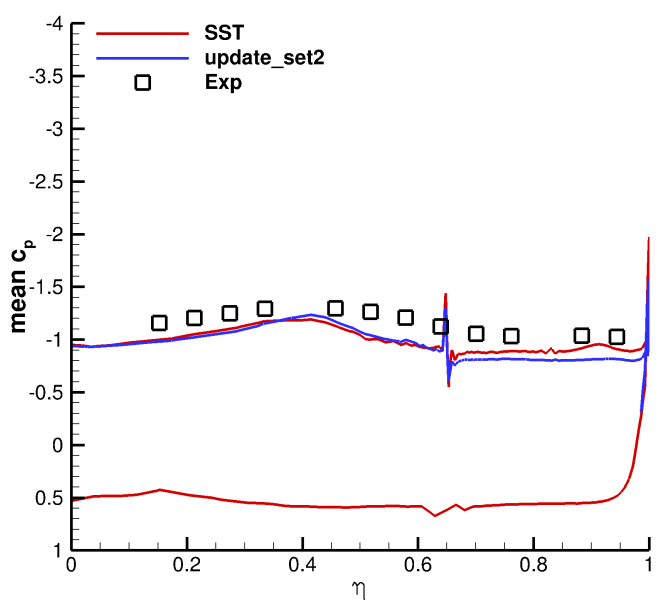

(c) $x / c_{r}=\mathbf{2 6 . 6 6 \%}$

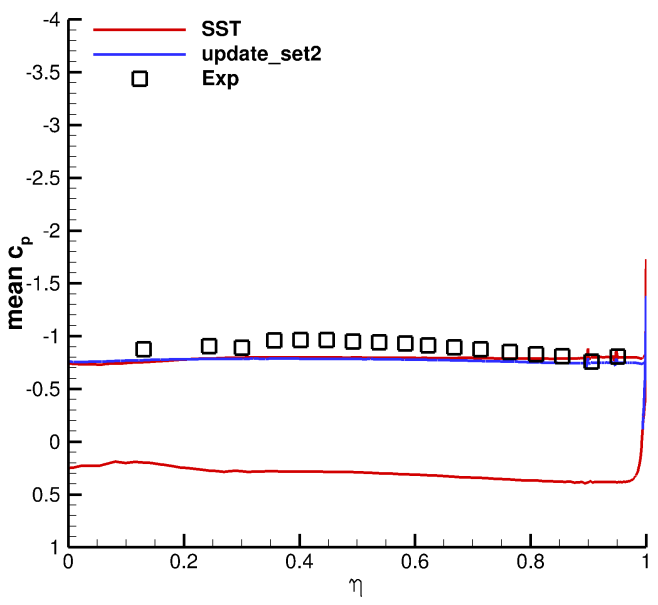

(e) $x / c_{r}=61.33 \%$

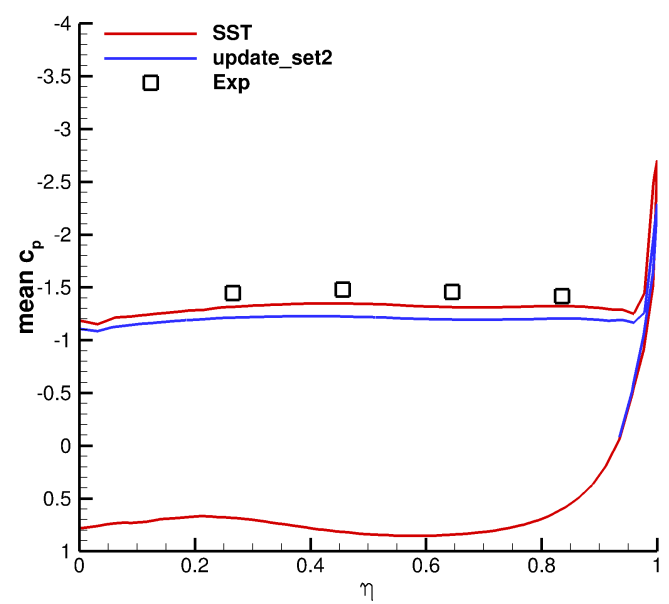

(b) $x / c_{r}=9.33 \%$

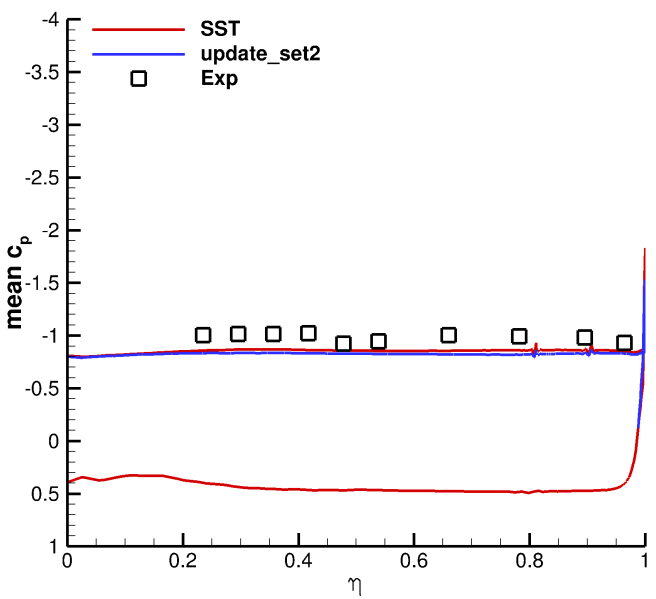

(d) $x / c_{r}=\mathbf{4 2 \%}$

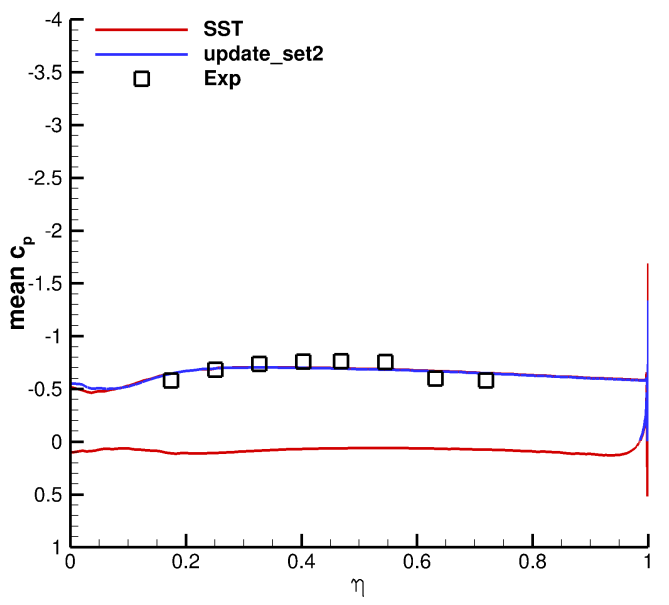

(f) $x / c_{r}=\mathbf{8 2 . 6 6 \%}$

Fig. 9 Calibration of spanwise surface pressure coefficient of Model 53 delta wing at $\alpha=28 \mathrm{deg}$ 


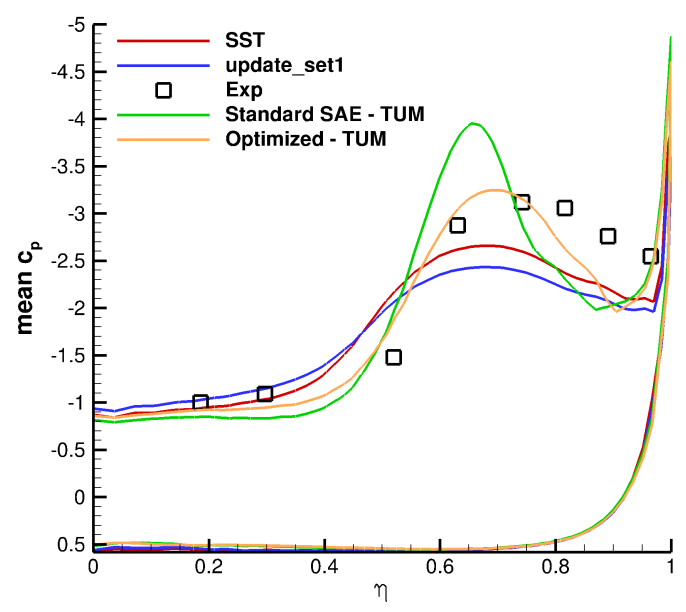

(a) $x / c_{r}=13.33 \%$

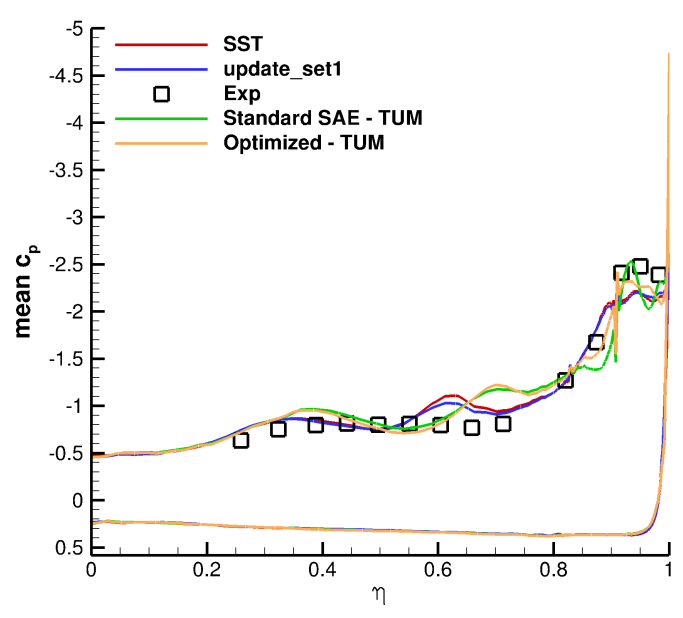

(c) $x / c_{r}=\mathbf{4 4 . 6 6 \%}$

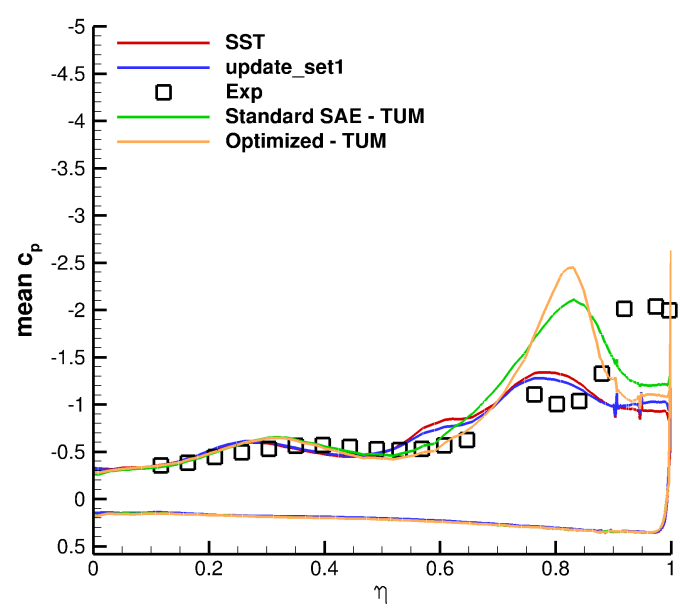

(e) $x / c_{r}=\mathbf{6 2 . 6 6 \%}$

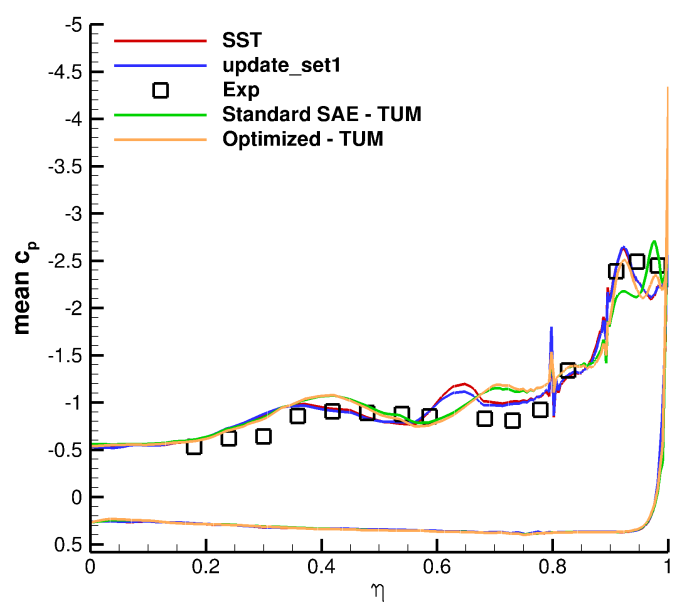

(b) $x / c_{r}=\mathbf{4 0 \%}$

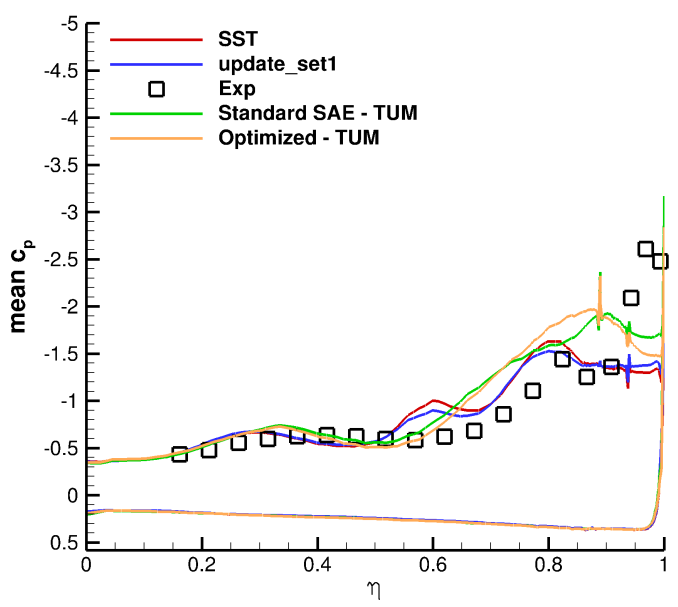

(d) $x / c_{r}=\mathbf{5 7 . 3 3 \%}$

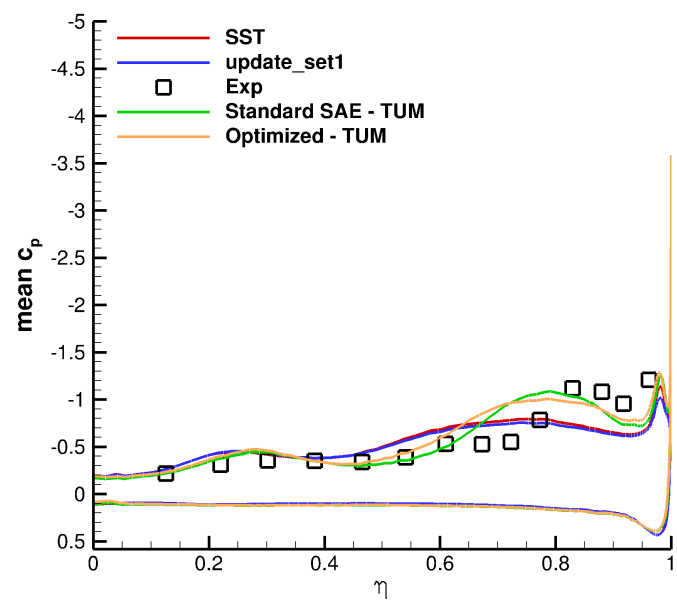

(f) $x / c_{r}=\mathbf{7 8 . 6 6 \%}$

Fig. 10 Validation of spanwise surface pressure coefficient of Model 56 delta wing at $\alpha=20 \mathrm{deg}$ 


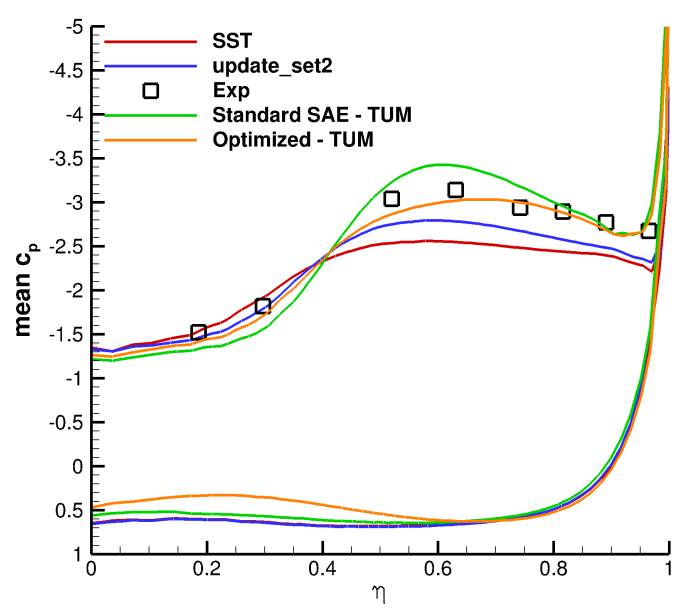

(a) $x / c_{r}=13.33 \%$

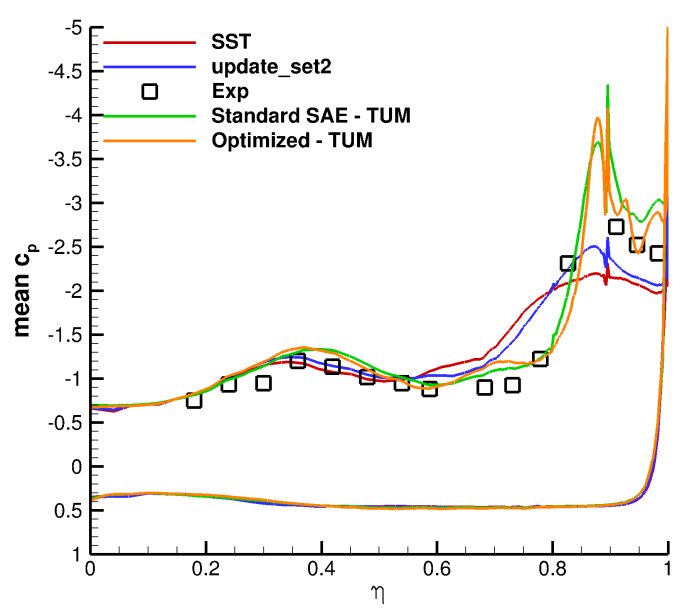

(c) $x / c_{r}=\mathbf{4 0 \%}$

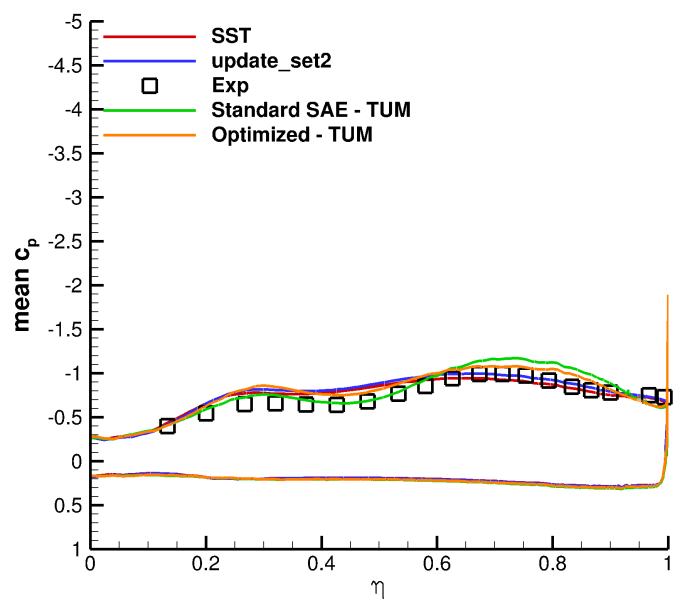

(e) $x / c_{r}=73.33 \%$

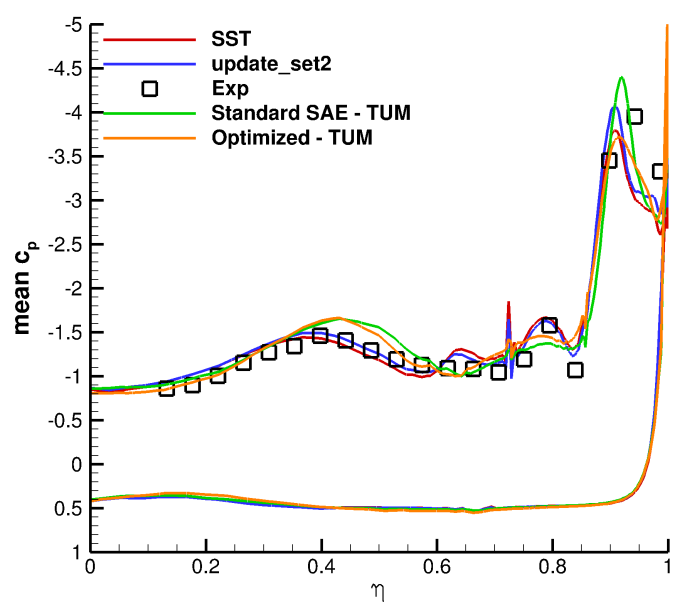

(b) $x / c_{r}=\mathbf{3 2 \%}$

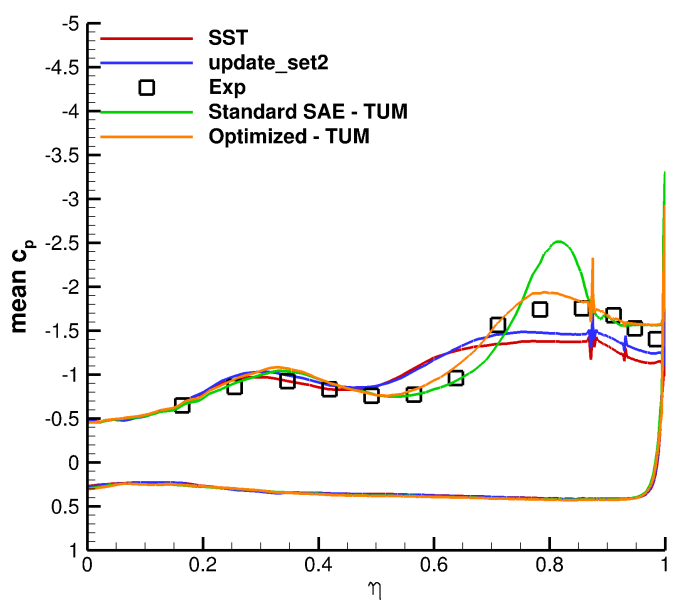

(d) $x / c_{r}=\mathbf{5 3 . 3 3 \%}$

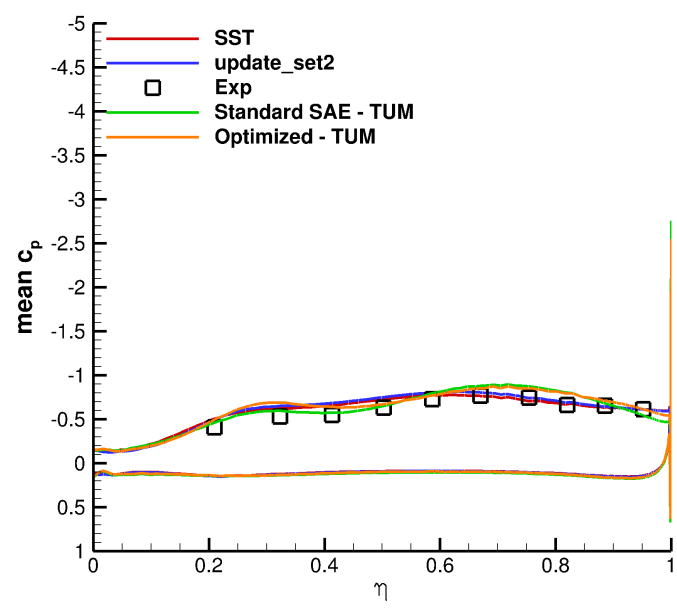

(f) $x / c_{r}=\mathbf{8 4 \%}$

Fig. 11 Validation of spanwise surface pressure coefficient of Model 56 delta wing at $\alpha=25 \mathrm{deg}$ 


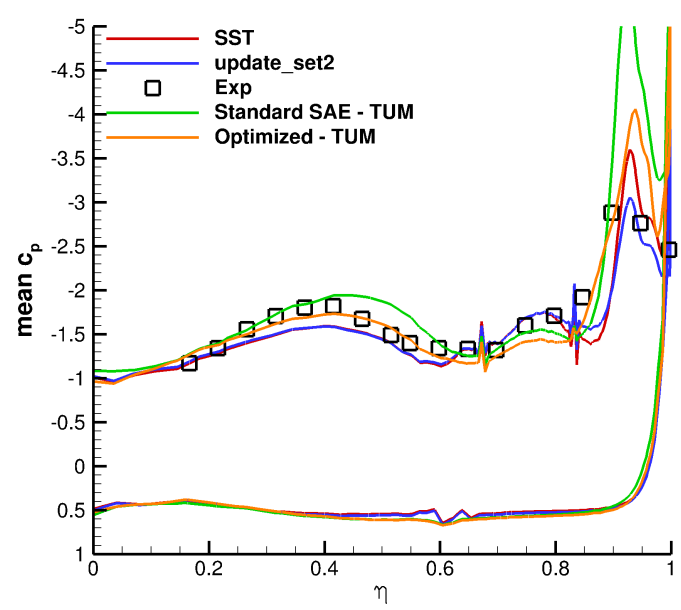

(a) $x / c_{r}=\mathbf{2 8 \%}$

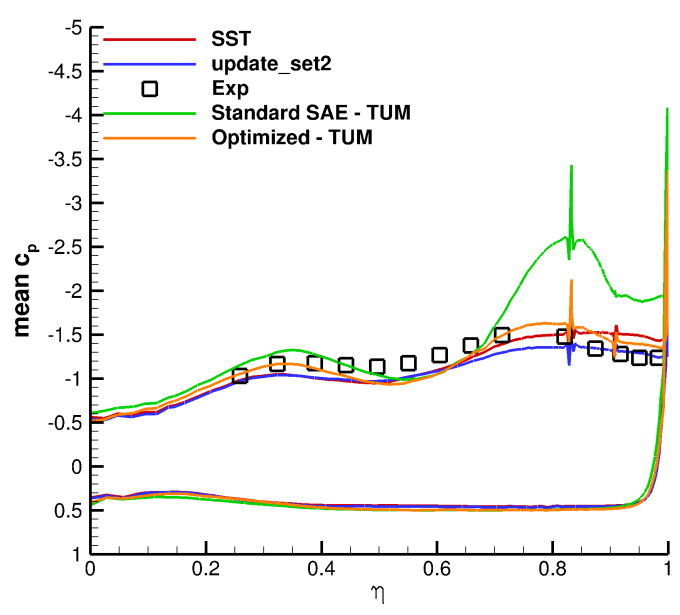

(c) $x / c_{r}=\mathbf{4 4 . 6 6 \%}$

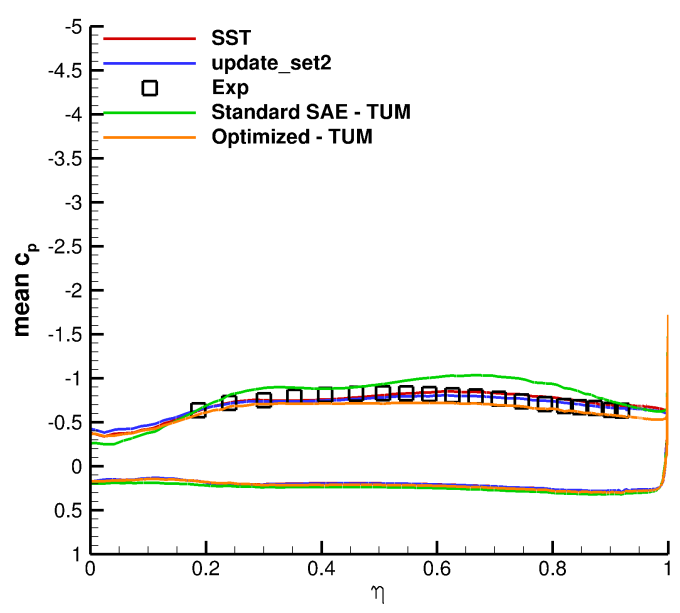

(e) $x / c_{r}=73.33 \%$

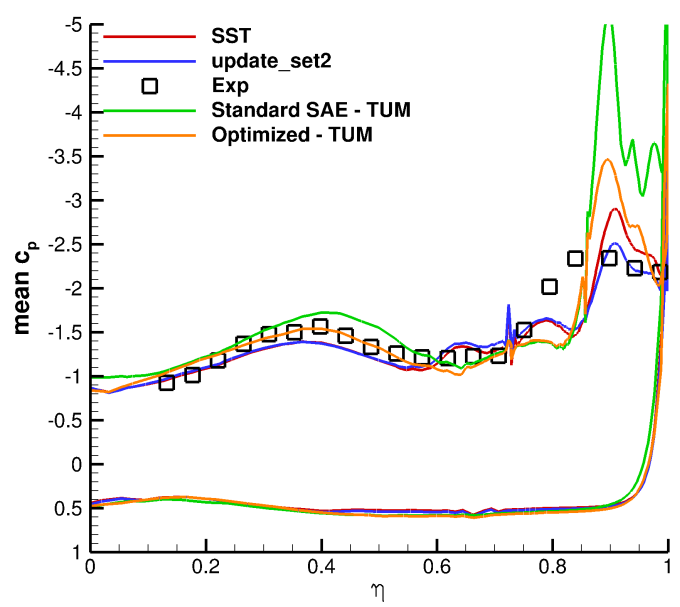

(b) $x / c_{r}=\mathbf{3 2 \%}$

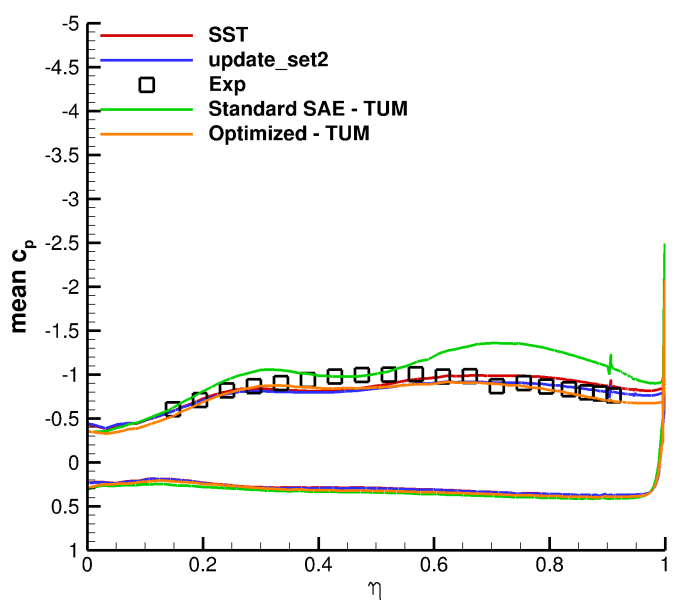

(d) $x / c_{r}=\mathbf{6 2 . 6 6 \%}$

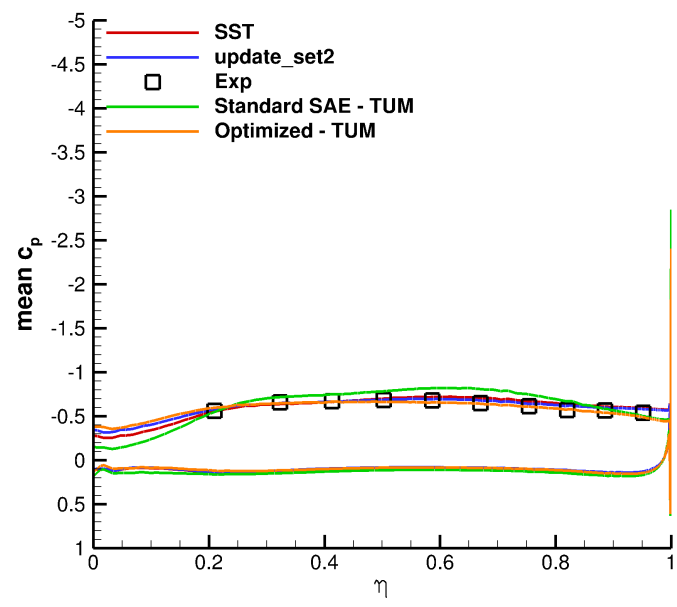

(f) $x / c_{r}=\mathbf{8 4 \%}$

Fig. 12 Validation of spanwise surface pressure coefficient of Model 56 delta wing at $\alpha=28 \mathrm{deg}$ 